This is a self-archived version of an original article. This version may differ from the original in pagination and typographic details.

Author(s): Gigli, Nicola; Pasqualetto, Enrico

Title: On the notion of parallel transport on RCD spaces

Year: 2020

Version: Published version

Copyright: (c) 2019 European Mathematical Society

Rights: In Copyright

Rights url: http://rightsstatements.org/page/lnC/1.0/?language=en

Please cite the original version:

Gigli, N., \& Pasqualetto, E. (2020). On the notion of parallel transport on RCD spaces. Revista Matematica Iberoamericana, 36(2), 571-609. https://doi.org/10.4171/rmi/1140 


\title{
On the notion of parallel transport on RCD spaces
}

\author{
Nicola Gigli and Enrico Pasqualetto
}

\begin{abstract}
We propose a general notion of parallel transport on RCD spaces, prove an unconditioned uniqueness result and existence under suitable assumptions on the space.
\end{abstract}

\section{Introduction}

More than ten years ago, Sturm [26], [27] and Lott-Villani [22] introduced the concept of lower Ricci curvature bounds for metric measure spaces. Their approach has been refined in [3] and [13] with the introduction of the class of metric measure spaces with Riemannian Ricci curvature bounded from below, RCD spaces in short, which is currently a very active research area. We refer to the surveys [29], [28] for an overview of the topic and detailed references.

Among the various recent contributions, of particular relevance for the current manuscript is the paper [15] by the first author, where a second order calculus has been built. In particular, on $\operatorname{RCD}(K, \infty)$ spaces the covariant derivative of vector fields is well-defined. Let us mention that in [15] 'vector field' is intended in the sense of abstract $L^{2}$-normed $L^{\infty}$-modules, and that in our previous paper [17] we showed that on $\operatorname{RCD}(K, N)$ spaces this abstract notion has a canonically more concrete counterpart described in terms of pointed-measured-Gromov-Hausdorff limits of rescaled spaces (this uses the rectifiability results obtained in [23] and in [21], [18]).

In the classical smooth Riemannian framework, covariant derivative and parallel transport are two closely related concepts, thus given the existence of covariant derivative on RCD spaces it is natural to ask: is there a notion of parallel transport in the same setting? In this paper we address this question, our main results being:

i) We provide a precise framework and give a rigorous meaning to the 'PDE' defining the parallel transport (see Definitions 3.19, 3.22 and 4.1).

ii) By the nature of our definition, norm-preservation and linearity of the parallel transport are easy to derive, and these in turn will give uniqueness (see Corollary 4.3).

Mathematics Subject Classification (2010): 30Lxx, 51Fxx.

Keywords: Parallel transport, RCD spaces. 
iii) On finite-dimensional RCD spaces satisfying a certain (strong) regularity property, we are able to show existence of the parallel transport (see Section 4.2). The regularity condition that we use is closely related to the existence of Sobolev vector fields with bounded covariant derivative (see Definition 4.9 for the precise assumption).

We believe that in fact the parallel transport exists on any RCD space, but we are currently unable to get the full proof. An insight on why this should not be too easy to prove is the following: on a space where the parallel transport exists, the dimension of the tangent module must be constant (see Theorem 4.8), and thanks to the aforementioned paper [17] this would in turn imply that the dimension of the pmGH-limits of rescaled spaces is constant. This very same result has been extremely elusive even in the context of Ricci-limit spaces and has been obtained only relatively recently by Colding-Naber in [8], while the constant dimension of all finite-dimensional RCD spaces has been proven by Bruè-Semola in [5]. Therefore it would be perhaps too optimistic to hope that the language proposed in [15] and here can easily imply such 'constant dimension' result. In this direction, we remark that the assumptions we put in order to obtain existence of the parallel transport are rather ad hoc and not really interesting from a geometric perspective: as pointed out by Lemma 4.12 below (see also Remark 4.13), they force the space to behave as a manifold of class $C^{1,1}$, in a sense. The intent with our existence result for this 'toy case' is just to show that the approach we propose is non-void. In the work-in-progress [7], we are investigating the same problem (together with E. Caputo) for more interesting geometric structures, namely finitedimensional Alexandrov spaces or, more generally, non-collapsed RCD spaces. The latter - called $\operatorname{ncRCD}(K, N)$ spaces for short - have been proposed in [9], and are characterised by the fact that the reference measure is given by the $N$-dimensional Hausdorff measure $\mathcal{H}^{N}$. The advantage of attacking the existence problem in this framework is that ncRCD spaces are Ahlfors regular, whence the Lusin-Lipschitz regularity results for regular Lagrangian flows that have been obtained in [6] seem to be helpful in providing existence of the parallel transport.

Let us also mention that in the appendix (see Theorem A.2) we prove that

iv) any $\operatorname{RCD}(K, \infty)$ space admits a basis of the tangent module made of vectors that belong to the space $W_{C}^{1,2}(T \mathrm{X})$ of Sobolev vector fields.

That is: if we relax the condition of 'bounded covariant derivative' present in (iii) above into 'covariant derivative in $L^{2}$ ', then every RCD space meets the requirement.

Let us now briefly describe our approach. The crucial idea is that we do not study the problem of parallel transport along a single Lipschitz curve, but rather we study the problem along $\boldsymbol{\pi}$-a.e. curve, where $\boldsymbol{\pi}$ is a Borel probability measure on the space $\Gamma(X)$ of continuous curves such that

$\boldsymbol{\pi}$ is concentrated on equi-Lipschitz curves,

and for some $C>0$ we have $\left(\mathrm{e}_{t}\right)_{*} \boldsymbol{\pi} \leq C \mathfrak{m}$ for every $t \in[0,1]$,

where $\mathfrak{m}$ is the given reference measure on our RCD space $X$ and $e_{t}: \Gamma(X) \rightarrow X$ is the evaluation map defined by $\mathrm{e}_{t}(\gamma):=\gamma_{t}$. Measures $\pi$ of this sort are a special 
case of so-called test plans introduced in [2]; these can be used to define Sobolev functions on metric measure spaces by a kind of duality argument. The advantage of working with these plans rather than with single curves is that they are naturally linked to Sobolev calculus and thus also to all the functional-analytic machinery built in $[15]$.

Let us now pretend, for the sake of this introduction, that our space $\mathrm{X}$ is in fact a smooth Riemannian manifold. In this case a time dependent vector field $\left(v_{t}\right)$ along $\boldsymbol{\pi}$ is, roughly said, given by a choice, for $\boldsymbol{\pi}$-a.e. $\gamma$, of time dependent vector fields $\left(v_{t}^{\gamma}\right)$ on $\mathbf{X}$. Then we say that $\left(v_{t}\right)$ is a parallel transport along $\boldsymbol{\pi}$ provided for $\pi$-a.e. $\gamma$ the vector field $t \mapsto v_{t}^{\gamma}\left(\gamma_{t}\right)$ is a parallel transport along $\gamma$. This happens if and only if

$$
\text { for } \pi \text {-a.e. } \gamma \text { we have } \partial_{t} v_{t}^{\gamma}+\nabla_{\gamma_{t}^{\prime}} v_{t}^{\gamma}=0 \quad \text { a.e. } t \text {. }
$$

A relevant part of our paper (the whole Chapter 3 ) is devoted to showing that the above PDE can be stated even in the non-smooth setting, the key point being that it is possible to define a closed operator acting on $L^{2}$ vector fields along $\boldsymbol{\pi}$ which plays the role of $\left(\partial_{t}+\nabla_{\gamma_{t}^{\prime}}\right)$, see Definitions 3.17, 3.19 and Proposition 3.20.

We conclude this introduction by describing two possible applications of the parallel transport theory for RCD spaces:

a) Petrunin proved in [24] that a certain 'geometric' notion of parallel transport exists along geodesics on Alexandrov spaces. Uniqueness for his construction is still an interesting open problem. On the other hand, in our 'functional analytic' approach uniqueness is trivial. It would be therefore interesting (and expected) to show that the two notions agree: among other things this would allow to obtain something new (i.e., uniqueness) for Petrunin's construction at least in some a.e. sense.

b) A more ambitious research direction is that of providing a more complete picture for what concerns a distributional approach to weak lower curvature bounds. Recall indeed that in [15] it has been introduced the Ricci curvature tensor in the RCD setting and that using the results in [4] one can see that, in a suitable sense, a metric measure space is $\operatorname{RCD}(K, \infty)$ if and only if such Ricci curvature is bounded from below by $K$. This provides a complete correspondence between the 'synthetic' Lott-Sturm-Villani approach [22], [26], [27] plus infinitesimal Hilbertianity [13] to lower Ricci curvature bounds and the 'distributional' approach based on a properly weakly defined Ricci tensor.

One could ask whether the same holds for lower sectional curvature bounds, where the standard definition based on triangle comparison would account for the 'synthetic' point of view. An important observation in this direction is the fact that on a RCD space one can give a meaning, in a very weak sense, to the full Riemann curvature tensor, and thus in particular also to the sectional curvature (see [16]). Recalling also Petrunin's [25] and Zhang-Zhu's [30] results it is therefore natural to ask: is it true that a RCD space is, as metric space, an Alexandrov space of curvature $\geq \kappa$ if and only if the sectional curvature in the above sense is bounded from below by $\kappa$ ? Shortly said, to answer this question one should prove 
Toponogov's theorem on RCD spaces and a key step to do this would be to prove the formula for the second variation of the energy of a curve. In turn, to obtain sharp estimates out of the variation of the energy it is crucial to have at disposal the parallel transport in order to have sufficient freedom in building vector fields along the geodesics we are varying.

In other words, having a good understanding of the parallel transport is a necessary (but far from sufficient) ingredient for both tightening the link between lower Ricci and lower sectional curvature bounds in the non-smooth setting and for providing a 'distributional' approach to the latter.

\section{Some basic notions}

To keep the presentation short, we shall assume the reader familiar with the language proposed in [15] (see also [14]).

\subsection{Curves in Banach spaces}

We recall here some basic results about measurability and integration of Banachvalued maps of a single variable $t \in[0,1]$. A detailed discussion about this topic can be found e.g. in [10].

Let $\mathbb{B}$ be a fixed Banach space. We will denote by $\mathbb{B}^{\prime}$ its dual space. A simple function is any map $y:[0,1] \rightarrow \mathbb{B}$ that can be written in the form

$$
y=\sum_{i=1}^{k} \chi_{E_{i}} v_{i}, \quad \text { for some } E_{1}, \ldots, E_{k} \in \mathscr{B}([0,1]) \text { and } v_{1}, \ldots, v_{k} \in \mathbb{B},
$$

where for any topological space $\mathrm{X}$ we denote by $\mathscr{B}(\mathrm{X})$ the set of Borel subsets of $\mathrm{X}$. A map $y:[0,1] \rightarrow \mathbb{B}$ is said to be strongly measurable provided there exists a sequence $\left(y_{n}\right)_{n}$ of simple functions $y_{n}:[0,1] \rightarrow \mathbb{B}$ such that $\lim _{n}\left\|y_{n}(t)-y(t)\right\|_{\mathbb{B}}=0$ for $\mathcal{L}^{1}$-a.e. $t \in[0,1]$, while it is said to be weakly measurable provided $[0,1] \ni t \mapsto$ $\omega(y(t)) \in \mathbb{R}$ is a Borel map for every $\omega \in \mathbb{B}^{\prime}$. It directly follows from the very definition that linear combinations of strongly (resp. weakly) measurable functions are strongly (resp. weakly) measurable. Moreover, if a map $y:[0,1] \rightarrow \mathbb{B}$ is strongly measurable, then the function $[0,1] \ni t \mapsto\|y(t)\|_{\mathbb{B}} \in \mathbb{R}$ is Borel.

The relation between the strongly measurable functions and the weakly measurable ones is fully described by a theorem of Pettis, which states that a function $y:[0,1] \rightarrow \mathbb{B}$ is strongly measurable if and only if it is weakly measurable and there exists a Borel set $N \subseteq[0,1]$ of null $\mathcal{L}^{1}$-measure such that $y([0,1] \backslash N)$ is a separable subset of $\mathbb{B}$.

We now describe how to define $\mathbb{B}$-valued integrals, the so-called Bochner integrals. First of all, given a simple function $y:[0,1] \rightarrow \mathbb{B}$, written as $y=\sum_{i=1}^{k} \chi_{E_{i}} v_{i}$, we define

$$
\int_{0}^{1} y(t) \mathrm{d} t:=\sum_{i=1}^{k} \mathcal{L}^{1}\left(E_{i}\right) v_{i} \in \mathbb{B} .
$$

It can be readily checked that this definition does not depend on the particular way of expressing the function $y$. 
Further, we say that any strongly measurable function $y:[0,1] \rightarrow \mathbb{B}$ is Bochner integrable provided there exists a sequence $\left(y_{n}\right)_{n}$ of simple functions $y_{n}:[0,1] \rightarrow \mathbb{B}$ such that $\lim _{n} \int_{0}^{1}\left\|y_{n}(t)-y(t)\right\|_{\mathbb{B}} \mathrm{d} t=0$. In particular, the sequence $\left(\int_{0}^{1} y_{n}(t) \mathrm{d} t\right)_{n}$ $\subseteq \mathbb{B}$ is Cauchy, so that it makes sense to define

$$
\int_{0}^{1} y(t) \mathrm{d} t:=\lim _{n \rightarrow \infty} \int_{0}^{1} y_{n}(t) \mathrm{d} t \in \mathbb{B} .
$$

It turns out that the value of $\int_{0}^{1} y(t) \mathrm{d} t$ just defined is independent of the approximating simple functions $\left(y_{n}\right)_{n}$ and that it satisfies the fundamental inequality

$$
\left\|\int_{0}^{1} y(t) \mathrm{d} t\right\|_{\mathbb{B}} \leq \int_{0}^{1}\|y(t)\|_{\mathbb{B}} \mathrm{d} t .
$$

An alternative characterisation of the $\mathbb{B}$-valued integrable maps is given by the following theorem, which is due to Bochner: a strongly measurable function $y:[0,1] \rightarrow \mathbb{B}$ is Bochner integrable if and only if it satisfies $\int_{0}^{1}\|y(t)\|_{\mathbb{B}} \mathrm{d} t<+\infty$.

The previous result naturally leads to the notion of $\mathbb{B}$-valued $L^{p}$ space: given $p \in[1, \infty]$, we define $L^{p}([0,1], \mathbb{B})$ as the space of all (equivalence classes of) those strongly measurable maps $y:[0,1] \rightarrow \mathbb{B}$ for which the quantity $\|y\|_{L^{p}([0,1], \mathbb{B})}$ is finite, where

$$
\|y\|_{L^{p}([0,1], \mathbb{B})}:= \begin{cases}\left(\int_{0}^{1}\|y(t)\|_{\mathbb{B}}^{p} \mathrm{~d} t\right)^{1 / p} & \text { if } p<\infty, \\ \underset{t \in[0,1]}{\operatorname{ess} \sup _{t}\|y(t)\|_{\mathbb{B}}} & \text { if } p=\infty .\end{cases}
$$

Hence $L^{p}([0,1], \mathbb{B})$ itself is a Banach space, for any $p \in[1, \infty]$.

Definition 2.1 (Vector-valued Sobolev/absolutely continuous maps). Let $p \in$ $[1, \infty]$. The space $W^{1, p}([0,1], \mathbb{B})$ consists of those curves $y \in L^{p}([0,1], \mathbb{B})$ for which there is $y^{\prime} \in L^{p}([0,1], \mathbb{B})$ such that

$$
\int_{0}^{1} \varphi^{\prime}(t) y(t) \mathrm{d} t=-\int_{0}^{1} \varphi(t) y^{\prime}(t) \mathrm{d} t, \quad \forall \varphi \in C_{c}^{\infty}(0,1) .
$$

It is equipped with the norm

$$
\|y\|_{W^{1, p}([0,1], \mathbb{B})}:=\left(\|y\|_{L^{p}([0,1], \mathbb{B})}^{p}+\left\|y^{\prime}\right\|_{L^{p}([0,1], \mathbb{B})}^{p}\right)^{1 / p} .
$$

The space $\operatorname{AC}^{p}([0,1], \mathbb{B})$ consists of those curves $y:[0,1] \rightarrow \mathbb{B}$ for which there is $f \in L^{p}(0,1)$ such that

$$
\|y(s)-y(t)\|_{\mathbb{B}} \leq \int_{t}^{s} f(r) \mathrm{d} r, \quad \forall t, s \in[0,1], t \leq s .
$$

Proposition 2.2 (Absolutely continuous representative). Let $y \in W^{1, p}([0,1], \mathbb{B})$. Then there exists a unique $\tilde{y} \in \mathrm{AC}^{p}([0,1], \mathbb{B})$ such that $y(t)=\tilde{y}(t)$ for a.e. $t$. Moreover, such $\tilde{y}$ satisfies

$$
\tilde{y}(s)-\tilde{y}(t)=\int_{t}^{s} y^{\prime}(r) \mathrm{d} r, \quad \forall t, s \in[0,1], t<s .
$$


Proof. The curve $t \mapsto z(t):=\int_{0}^{t} y^{\prime}(s) \mathrm{d} s$ belongs to $\mathrm{AC}^{p} \cap W^{1, p}([0,1], \mathbb{B})$ and thus in particular the curve $t \mapsto y(t)-z(t)$ belongs to $W^{1, p}([0,1], \mathbb{B})$ and, obviously, has derivative a.e. equal to 0 . Hence to conclude it is sufficient to show that any such curve is a.e. constant. This follows noticing that for any $\ell \in \mathbb{B}^{\prime}$ the map $t \mapsto \ell(y(t)-z(t))$ is in $W^{1, p}([0,1])$ (by direct verification) and has derivative a.e. equal to 0 .

Proposition 2.3 (Characterization of curves in the space $W^{1, p}([0,1], \mathbb{B})$ ). Let $y, z \in L^{p}([0,1], \mathbb{B})$. Then $y \in W^{1, p}([0,1], \mathbb{B})$ and $z=y^{\prime}$ if and only if for some dense set $D \subset \mathbb{B}^{\prime}$ we have that $\ell \circ y \in W^{1,1}([0,1])$ with $(\ell \circ y)^{\prime}=\ell \circ z$ a.e. for every $\ell \in D$.

In particular, if $\mathbb{B}=L^{\tilde{p}}(\mu)$ for some Radon measure $\mu$, then $y \in W^{1, p}([0,1]$, $\left.L^{\tilde{p}}(\mu)\right)$ and $z=y^{\prime}$ if and only if for every Borel set $E$ we have that $t \mapsto \int_{E} y(t) \mathrm{d} \mu$ is in $W^{1,1}(0,1)$ with derivative given by $t \mapsto \int_{E} z(t) \mathrm{d} \mu$.

Proof. By assumption and using the fact that the Bochner integral commutes with the application of $\ell$, we have that

$$
\ell\left(\int_{0}^{1} \varphi^{\prime}(t) y(t) \mathrm{d} t\right)=\ell\left(-\int_{0}^{1} \varphi(t) z(t) \mathrm{d} t\right) \quad \forall \varphi \in C_{c}^{\infty}(0,1)
$$

for every $\ell \in D$. The conclusion follows by the density of $D$ in $\mathbb{B}^{\prime}$.

For the second claim just observe that the linear span of the set of characteristic functions of Borel sets is dense in $L^{\tilde{q}}(\mu) \sim\left(L^{\tilde{p}}(\mu)\right)^{\prime}$.

It is important to underline that in general absolute continuity does not imply a.e. differentiability: for instance, the curve $[0,1] \ni t \mapsto \chi_{[0, t]} \in L^{1}(0,1)$ is absolutely continuous (as it is an isometry), but it is nowhere differentiable. The a.e. differentiability of absolutely continuous curves has to do with the so-called RadonNikodym property of the target Banach space. A sufficient condition for this implication to hold is given in the next theorem.

Theorem 2.4. Let $\mathbb{B}$ be a reflexive Banach space, $p \in[1, \infty]$ and $y \in \mathrm{AC}^{p}([0,1], \mathbb{B})$. Then for a.e. $t \in[0,1]$ the limit of $(y(t+h)-y(t)) / h$ as $h \rightarrow 0$ exists in $\mathbb{B}$.

In particular, $\mathrm{AC}^{p}([0,1], \mathbb{B}) \sim W^{1, p}([0,1], \mathbb{B})$, i.e., every absolutely continuous curve is the (only) continuous representative of a curve in $W^{1, p}([0,1], \mathbb{B})$.

Proof. The first statement - namely, the a.e. differentiability of any element of $\operatorname{AC}^{p}([0,1], \mathbb{B})$ - is well known. For the second statement, fix any $y \in \mathrm{AC}^{p}([0,1], \mathbb{B})$ and $\ell \in \mathbb{B}^{\prime}$. Let us set $z(t):=\lim _{h \rightarrow 0}(y(t+h)-y(t)) / h$ for a.e. $t \in[0,1]$. Notice that $z \in L^{p}([0,1], \mathbb{B})$. Trivially, the function $\ell \circ y$ is absolutely continuous and a.e. differentiable, with derivative $\ell \circ z$, whence by using the dominated convergence theorem one can conclude that $\ell \circ y \in W^{1,1}(0,1)$ and $(\ell \circ y)^{\prime}=\ell \circ z$ in the a.e. sense. Thanks to Proposition 2.3, this proves that (the equivalence class of) $y$ belongs to $W^{1, p}([0,1], \mathbb{B})$, as required. 
Given any Banach space $\mathbb{B}$, we shall denote by $\operatorname{End}(\mathbb{B})$ the space of all linear and continuous maps of $\mathbb{B}$ to itself, which is a Banach space if endowed with the operator norm.

The space $\Gamma(\mathbb{B}):=C([0,1], \mathbb{B})$ is a Banach space with respect to the norm $\|\cdot\|_{\Gamma(\mathbb{B})}$, given by $\|y\|_{\Gamma(\mathbb{B})}:=\max \left\{\left\|y_{t}\right\|_{\mathbb{B}}: t \in[0,1]\right\}$ for every $y \in \Gamma(\mathbb{B})$.

Theorem 2.5 (Integral solutions to vector-valued linear ODEs). Let $\mathbb{B}$ be a $\mathrm{Ba}$ nach space. Let $z \in \Gamma(\mathbb{B})$. Let $\lambda:[0,1] \rightarrow \operatorname{End}(\mathbb{B})$ be a bounded function, i.e., there exists $c>0$ such that $\|\lambda(t)\|_{\operatorname{End}(\mathbb{B})} \leq c$ for every $t \in[0,1]$. Assume that $[0,1] \ni t \mapsto \lambda(t) v \in \mathbb{B}$ is strongly measurable for every $v \in \mathbb{B}$. Then there exists a unique curve $y \in \Gamma(\mathbb{B})$ such that

$$
y(t)=z(t)+\int_{0}^{t} \lambda(s) y(s) \mathrm{d} s \quad \text { for every } t \in[0,1] .
$$

Moreover, the solution y satisfies $\|y\|_{\Gamma(\mathbb{B})} \leq e^{c}\|z\|_{\Gamma(\mathbb{B})}$.

Proof. Given any simple function $t \mapsto y_{t}=\sum_{i=1}^{k} \chi_{A_{i}}(t) v_{i}$, with $A_{1}, \ldots, A_{k} \in$ $\mathscr{B}([0,1])$ and $v_{1}, \ldots, v_{k} \in \mathbb{B}$, we have that $t \mapsto \lambda(t) y_{t}=\sum_{i=1}^{k} \chi_{A_{i}}(t) \lambda(t) v_{i}$ is strongly measurable by hypothesis on $\lambda$. Now fix $y \in \Gamma(\mathbb{B})$. In particular, $y:[0,1] \rightarrow \mathbb{B}$ is strongly measurable, hence there exists a sequence $\left(y^{k}\right)_{k}$ of simple functions $y^{k}:[0,1] \rightarrow \mathbb{B}$ such that $\lim _{k}\left\|y_{t}^{k}-y_{t}\right\|_{\mathbb{B}}=0$ holds for $\mathcal{L}^{1}$-a.e. $t \in[0,1]$. This grants that $\left\|\lambda(t) y_{t}^{k}-\lambda(t) y_{t}\right\|_{\mathbb{B}} \leq c\left\|y_{t}^{k}-y_{t}\right\|_{\mathbb{B}} \stackrel{k}{\rightarrow} 0$ is satisfied for $\mathcal{L}^{1}$-a.e. $t \in[0,1]$, thus accordingly the map $t \mapsto \lambda(t) y_{t}$ is strongly measurable as pointwise limit of strongly measurable functions. Moreover, since $\left\|\lambda(t) y_{t}\right\|_{\mathbb{B}} \leq c\|y\|_{\Gamma(\mathbb{B})}$ for all $t \in[0,1]$, one has that $t \mapsto \lambda(t) y_{t}$ actually belongs to $L^{\infty}([0,1], \mathbb{B})$. Therefore it makes sense to define the function $\Lambda y:[0,1] \rightarrow \mathbb{B}$ as $(\Lambda y)(t):=\int_{0}^{t} \lambda(s) y_{s} \mathrm{~d} s$ for every $t \in[0,1]$. Note that

$$
\left\|\Lambda y\left(t_{1}\right)-\Lambda y\left(t_{0}\right)\right\|_{\mathbb{B}} \leq c\|y\|_{\Gamma(\mathbb{B})}\left(t_{1}-t_{0}\right) \quad \text { for every } t_{0}, t_{1} \in[0,1] \text { with } t_{0}<t_{1} .
$$

Then $\Lambda y$ is Lipschitz with $\operatorname{Lip}(\Lambda y) \leq c\|y\|_{\Gamma(\mathbb{B})}$, so in particular $\Lambda y \in \Gamma(\mathbb{B})$. By plugging $t_{1}=t$ and $t_{0}=0$ into (2.3), we deduce that $\|\Lambda y(t)\|_{\mathbb{B}} \leq c\|y\|_{\Gamma(\mathbb{B})} t$ for all $t \in[0,1]$ and accordingly that $\|\Lambda y\|_{\Gamma(\mathbb{B})} \leq c\|y\|_{\Gamma(\mathbb{B})}$. This guarantees that the mapping $\Lambda: \Gamma(\mathbb{B}) \rightarrow \Gamma(\mathbb{B})$ is linear and continuous, with $\|\Lambda\|_{\operatorname{End}(\Gamma(\mathbb{B}))} \leq c$. Now observe that

$$
y \in \Gamma(\mathbb{B}) \text { satisfies }(2.2) \Longleftrightarrow\left(\operatorname{Id}_{\Gamma(\mathbb{B})}-\Lambda\right)(y)=z .
$$

Standard verifications show that the iterated operator $\Lambda^{n}$ satisfies $\left\|\Lambda^{n}\right\|_{\operatorname{End}(\Gamma(\mathbb{B}))} \leq$ $c^{n} / n$ !. Hence $\operatorname{Id}_{\Gamma(\mathbb{B})}-\Lambda$ is invertible and the operator norm of $\left(\operatorname{Id}_{\Gamma(\mathbb{B})}-\Lambda\right)^{-1}=$ $\sum_{n=0}^{\infty} \Lambda^{n}$ is bounded above by $e^{c}$. In light of (2.4), we finally conclude that there exists a unique curve $y \in \Gamma(\mathbb{B})$ fulfilling $(2.2)$, namely $y:=\left(\operatorname{Id}_{\Gamma(\mathbb{B})}-\Lambda\right)^{-1}(z)$, which also satisfies $\|y\|_{\Gamma(\mathbb{B})} \leq e^{c}\|z\|_{\Gamma(\mathbb{B})}$.

The following result can be readily achieved as a consequence of Theorems 2.4 and 2.5 . 
Corollary 2.6 (Differential solutions to vector-valued linear ODEs). Fix a reflexive Banach space $\mathbb{B}$. Let $\bar{y} \in \mathbb{B}$. Let $\lambda:[0,1] \rightarrow \operatorname{End}(\mathbb{B})$ be a bounded function. Suppose that the map $[0,1] \ni t \mapsto \lambda(t) v \in \mathbb{B}$ is strongly measurable for every $v \in \mathbb{B}$. Then there exists a unique curve $y \in \operatorname{LIP}([0,1], \mathbb{B})$ such that

$$
\left\{\begin{array}{l}
y^{\prime}(t)=\lambda(t) y(t) \quad \text { for } \mathcal{L}^{1} \text {-a.e. } t \in[0,1], \\
y(0)=\bar{y} .
\end{array}\right.
$$

Moreover, the solution y satisfies $\|y\|_{\Gamma(\mathbb{B})} \leq e^{c}\|\bar{y}\|_{\mathbb{B}}$, where $c:=\max _{t \in[0,1]}\|\lambda(t)\|_{\operatorname{End}(\mathbb{B})}$.

\subsection{Pullback of an $L^{0}$-normed module}

The aim of this subsection is to introduce the concept of pullback of an $L^{0}$-normed module and to study its main properties. The following definitions and results mimic and follow by the analogous ones for $L^{p}$-normed modules, which are treated in [15], Subsection 1.6; a digression similar to the one below has been done in [20].

Theorem 2.7. Let $\left(\mathrm{X}, \mathcal{A}_{\mathrm{X}}, \mathfrak{m}_{\mathrm{X}}\right),\left(\mathrm{Y}, \mathcal{A}_{\mathrm{Y}}, \mathfrak{m}_{\mathrm{Y}}\right)$ be $\sigma$-finite measured spaces. Let $\varphi: \mathrm{X} \rightarrow \mathrm{Y}$ be a map of bounded compression (i.e., $\varphi_{*} \mathfrak{m}_{\mathrm{X}} \leq C \mathfrak{m}_{\mathrm{Y}}$ for some $C>0$ ). Let $\mathscr{M}^{0}$ be an $L^{0}\left(\mathfrak{m}_{\mathrm{Y}}\right)$-normed module. Then there exists (up to unique isomorphism) a unique couple $\left(\mathscr{N}^{0}, \mathrm{~T}\right)$, where $\mathscr{N}^{0}$ is an $L^{0}\left(\mathfrak{m}_{\mathrm{X}}\right)$-normed module and $\mathrm{T}: \mathscr{M}^{0} \rightarrow \mathscr{N}^{0}$ is a linear map, such that

(i) $|\mathrm{T} v|=|v| \circ \varphi$ holds $\mathfrak{m}_{\mathrm{X}}$-a.e. in $\mathrm{X}$, for every $v \in \mathscr{M}^{0}$,

(ii) the set of all the elements of the form $\sum_{i=1}^{n} \chi_{A_{i}} \mathrm{~T} v_{i}$, with $\left(A_{i}\right)_{i=1}^{n} \subseteq \mathcal{A}_{\mathrm{X}}$ partition of $\mathrm{X}$ and $v_{1}, \ldots, v_{n} \in \mathscr{M}^{0}$, is dense in $\mathscr{N}^{0}$.

Namely, if two couples $\left(\mathscr{N}_{1}^{0}, \mathrm{~T}_{1}\right)$ and $\left(\mathscr{N}_{2}^{0}, \mathrm{~T}_{2}\right)$ as above fulfill both (i) and (ii), then there exists a unique module isomorphism $\Phi: \mathscr{N}_{1}^{0} \rightarrow \mathscr{N}_{2}^{0}$ such that the diagram



is commutative.

Proof. Existence. Fix $p \in[1, \infty)$ and let $\mathscr{M}:=\left\{v \in \mathscr{M}^{0}:|v| \in L^{p}\left(\mathfrak{m}_{\mathrm{Y}}\right)\right\}$. Hence $\mathscr{M}$ is an $L^{p}\left(\mathfrak{m}_{Y}\right)$-normed module and $\mathscr{M}^{0}$ is the $L^{0}$-completion of $\mathscr{M}$. Define $\mathscr{N}^{0}:=\left(\varphi^{*} \mathscr{M}\right)^{0}$. We construct the map $\mathrm{T}$ in the following way: since the space $\mathscr{M}$ is dense in $\mathscr{M}^{0}$ and $\varphi^{*} \mathscr{M}$ is continuously embedded into $\mathscr{N}^{0}$, the map $\varphi^{*}: \mathscr{M} \rightarrow \varphi^{*} \mathscr{M}$ can be uniquely extended to a linear continuous function $\mathrm{T}: \mathscr{M}^{0} \rightarrow \mathscr{N}^{0}$. Such function T also satisfies (i). Moreover, it is clear that the diagram

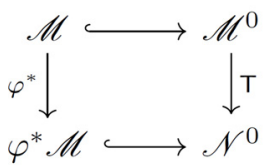


commutes. Therefore, since the set $\left\{\varphi^{*} v: v \in \mathscr{M}\right\}$ generates $\mathscr{N}^{0}$ as $L^{0}\left(\mathfrak{m}_{\mathrm{X}}\right)$ normed module, we have in particular that the set $\left\{\mathrm{T} v: v \in \mathscr{M}^{0}\right\}$ generates $\mathscr{N}^{0}$ as $L^{0}\left(\mathfrak{m}_{\mathrm{X}}\right)$-normed module, proving (ii).

Uniqueness. Let us choose $\left(\mathscr{N}_{1}^{0}, \mathrm{~T}_{1}\right),\left(\mathscr{N}_{2}^{0}, \mathrm{~T}_{2}\right)$ satisfying (i) and (ii). For $j=1,2$, denote by $V_{j}$ the set of $\sum_{i=1}^{n} \chi_{A_{i}} \mathrm{~T}_{j} v_{i}$ as in (ii). Then the unique $L^{0}\left(\mathfrak{m}_{\mathrm{X}}\right)$ linear map $\Psi: V_{1} \rightarrow V_{2}$, which satisfies $\Psi \circ \mathrm{T}_{1}=\mathrm{T}_{2}$, is necessarily given by $\Psi\left(\sum_{i=1}^{n} \chi_{A_{i}} \mathrm{\top}_{1} v_{i}\right)=\sum_{i=1}^{n} \chi_{A_{i}} \mathrm{~T}_{2} v_{i}$. By requiring the condition (i), we force the $\mathfrak{m}_{\mathrm{X}}$-a.e. equality

$$
\left|\sum_{i=1}^{n} \chi_{A_{i}} \mathrm{\top}_{2} v_{i}\right|=\sum_{i=1}^{n} \chi_{A_{i}}\left|v_{i}\right| \circ \varphi=\left|\sum_{i=1}^{n} \chi_{A_{i}} \mathrm{\top}_{1} v_{i}\right|,
$$

which shows that the map $\Psi$ is actually well-defined and continuous. There exists a unique module morphism $\Phi: \mathscr{N}_{1}^{0} \rightarrow \mathscr{N}_{2}^{0}$ that extends $\Psi$, by density of $V_{1}$ in $\mathscr{N}_{1}^{0}$. Such map $\Phi$ clearly satisfies $\Phi \circ \mathrm{T}_{1}=\mathrm{T}_{2}$. Finally, by interchanging the roles of $\mathscr{N}_{1}^{0}$ and $\mathscr{N}_{2}^{0}$, one can easily conclude that $\Phi$ is an isomorphism, getting the thesis.

Definition 2.8 (Pullback module). Any couple $\left(\mathscr{N}^{0}, \mathrm{~T}\right)$ that satisfies Theorem 2.7 will be unambiguously denoted by $\left(\varphi^{*} \mathscr{M}^{0}, \varphi^{*}\right)$. Moreover, we shall call $\varphi^{*} \mathscr{M}^{0}$ the pullback module of $\mathscr{M}^{0}$ and $\varphi^{*}$ the pullback map.

Proposition 2.9 (Universal property of the pullback). Let (X, $\mathcal{A}_{\mathrm{X}}, \mathfrak{m}_{\mathrm{X}}$ ) and $\left(\mathrm{Y}, \mathcal{A}_{\mathrm{Y}}, \mathfrak{m}_{\mathrm{Y}}\right)$ be $\sigma$-finite measured spaces. Let $\varphi: \mathrm{X} \rightarrow \mathrm{Y}$ be a map of bounded compression. Let $\mathscr{M}^{0}$ be an $L^{0}\left(\mathfrak{m}_{\mathrm{Y}}\right)$-normed module and let $\mathscr{N}^{0}$ be an $L^{0}\left(\mathfrak{m}_{\mathrm{X}}\right)$ normed module. Consider a linear operator $\mathrm{T}: \mathscr{M}^{0} \rightarrow \mathscr{N}^{0}$ such that

$$
|\mathrm{T} v| \leq \ell|v| \circ \varphi \quad \mathfrak{m}_{\mathrm{X}} \text {-a.e. in } \mathrm{X}, \quad \text { for every } v \in \mathscr{M}^{0},
$$

for a suitable map $\ell \in L^{0}\left(\mathfrak{m}_{\mathrm{X}}\right)$. Then there exists a unique $L^{0}\left(\mathfrak{m}_{\mathrm{X}}\right)$-linear and continuous operator $\widehat{\mathrm{T}}: \varphi^{*} \mathscr{M}^{0} \rightarrow \mathscr{N}^{0}$ such that $\widehat{\mathrm{T}} \circ \varphi^{*}=\mathrm{T}$ and

$$
|\widehat{\mathrm{T}} w| \leq \ell|w| \quad \mathfrak{m}_{\mathrm{X}} \text {-a.e. in } \mathrm{X}, \quad \text { for every } w \in \varphi^{*} \mathscr{M}^{0} .
$$

Proof. Denote by $V$ the set of all elements of the form $\sum_{i=1}^{n} \chi_{A_{i}} \varphi^{*} v_{i}$, with $A_{1}, \ldots, A_{n}$ $\in \mathcal{A}_{\mathrm{X}}$ partition of $\mathrm{X}$ and $v_{1}, \ldots, v_{n} \in \mathscr{M}^{0}$, so that $V$ is a dense linear subspace of $\varphi^{*} \mathscr{M}^{0}$ by property (ii) of Theorem 2.7. Any $L^{0}\left(\mathfrak{m}_{\mathrm{X}}\right)$-linear map $\widehat{\mathrm{T}}: \varphi^{*} \mathscr{M}^{0} \rightarrow \mathscr{N}^{0}$ with $\widehat{T} \circ \varphi^{*}=\mathrm{T}$ must satisfy

$$
\widehat{\mathrm{T}} w=\sum_{i=1}^{n} \chi_{A_{i}} \widehat{\mathrm{T}}\left(\varphi^{*} v_{i}\right)=\sum_{i=1}^{n} \chi_{A_{i}} \mathrm{\top} v_{i}, \quad \text { for } w=\sum_{i=1}^{n} \chi_{A_{i}} \varphi^{*} v_{i} \in V .
$$

Consider $\widehat{\mathrm{T}}: V \rightarrow \mathscr{N}^{0}$ defined as in (2.8), then (2.6) grants that

$$
\begin{aligned}
|\widehat{\mathrm{T}} w| & =\sum_{i=1}^{n} \chi_{A_{i}}\left|\mathrm{~T} v_{i}\right| \leq \ell \sum_{i=1}^{n} \chi_{A_{i}}\left|v_{i}\right| \circ \varphi \\
& =\ell \sum_{i=1}^{n} \chi_{A_{i}}\left|\varphi^{*} v_{i}\right|=\ell|w| \quad \text { holds } \mathfrak{m}_{\mathrm{X}} \text {-a.e. }
\end{aligned}
$$


which shows that $\widehat{\mathrm{T}}: V \rightarrow \mathscr{N}^{0}$ is well-defined (in the sense that $\widehat{\mathrm{T}} w$ depends only on $w$ and not on the way of representing it) and continuous. Therefore $\widehat{\mathrm{T}}$ can be uniquely extended to a linear continuous operator $\widehat{T}: \varphi^{*} \mathscr{M}^{0} \rightarrow \mathscr{N}^{0}$. We readily deduce from the definition (2.8) that the equality $f \widehat{\mathrm{T}} w=\widehat{\mathrm{T}}(f w)$ holds for $f: \mathrm{X} \rightarrow \mathbb{R}$ simple function, so that $\widehat{\mathrm{T}}$ can be shown to be $L^{0}\left(\mathfrak{m}_{\mathrm{X}}\right)$-linear by an approximation argument. Finally, it follows from (2.9) that the inequality (2.7) is satisfied for $w \in V$, whence (2.7) holds by density of $V$ in $\varphi^{*} \mathscr{M}^{0}$.

\subsection{Some properties of test plans}

For the sake of brevity, hereafter we shall use the notation $\mathcal{L}_{1}$ to indicate the 1-dimensional Lebesgue measure restricted to [0,1], namely

$$
\mathcal{L}_{1}:=\left.\mathcal{L}^{1}\right|_{[0,1]} .
$$

Let $\boldsymbol{\pi} \in \mathscr{P}(\Gamma(\mathrm{X}))$ be any fixed test plan on $\mathrm{X}$, whence $\left(\Gamma(\mathrm{X}), \mathrm{d}_{\Gamma(\mathrm{X})}, \boldsymbol{\pi}\right)$ is a metric measure space. Given that the map $\mathrm{e}_{t}$ is of bounded compression, it makes sense to consider the pullback module $\mathrm{e}_{t}^{*} L^{2}(T \mathrm{X})$. Observe that $\mathrm{e}_{t}^{*} L^{2}(T \mathrm{X})$ is a Hilbert module as soon as $(\mathrm{X}, \mathrm{d}, \mathfrak{m})$ is infinitesimally Hilbertian.

Remark 2.10. Let us define the map e: $\Gamma(\mathrm{X}) \times[0,1] \rightarrow \mathrm{X}$ as $\mathrm{e}(\gamma, t):=\gamma_{t}$ for every $\gamma \in \Gamma(\mathrm{X})$ and $t \in[0,1]$. It can be easily proved that the map e is continuous. This grants that, given any Borel map $f: \mathrm{X} \rightarrow \mathbb{R}$, the function $f \circ \mathrm{e}$ is Borel. Moreover, observe that

$$
\left(\boldsymbol{\pi} \times \mathcal{L}_{1}\right)\left(\mathrm{e}^{-1}(A)\right)=\int_{0}^{1} \boldsymbol{\pi}\left(\mathrm{e}_{t}^{-1}(A)\right) \mathrm{d} t \leq \mathrm{C}(\boldsymbol{\pi}) \mathfrak{m}(A) \quad \text { for every } A \in \mathscr{B}(\mathrm{X})
$$

by Fubini's theorem, in other words it holds that $\mathrm{e}_{*}\left(\boldsymbol{\pi} \times \mathcal{L}_{1}\right) \leq \mathrm{C}(\boldsymbol{\pi}) \mathfrak{m}$. Therefore one has that the composition $f \circ \mathrm{e} \in L^{0}\left(\boldsymbol{\pi} \times \mathcal{L}_{1}\right)$ is well-defined for any $f \in L^{0}(\mathfrak{m})$.

The following result belongs to the folklore of the theory; we provide a proof for completeness.

Theorem 2.11. Let $(\mathrm{X}, \mathrm{d}, \mathfrak{m})$ be a metric measure space. Let $\boldsymbol{\pi}$ be a test plan on $\mathrm{X}$. Then

(2.10) for every $f \in L^{1}(\mathfrak{m})$ the map $[0,1] \ni t \longmapsto f \circ \mathrm{e}_{t} \in L^{1}(\boldsymbol{\pi})$ is continuous.

In particular, the map $[0,1] \ni t \mapsto \int f \circ \mathrm{e}_{t} \mathrm{~d} \boldsymbol{\pi}$ is continuous for every $f \in L^{1}(\mathfrak{m})$.

Proof. First of all, we claim that

$$
\lim _{s \rightarrow t} \int\left|f \circ \mathrm{e}_{s}-f \circ \mathrm{e}_{t}\right| \mathrm{d} \boldsymbol{\pi}=0 \quad \text { if } f \in C_{b}(\mathrm{X}) \cap L^{1}(\mathfrak{m}) \text { and } t \in[0,1] .
$$

To prove it, note that $\left|f \circ \mathrm{e}_{s}-f \circ \mathrm{e}_{t}\right|(\gamma) \leq 2\|f\|_{L^{\infty}(\mathfrak{m})}$ for every $\gamma \in \Gamma(\mathrm{X})$ and $t, s$ $\in[0,1]$. Moreover, $\left|f\left(\gamma_{s}\right)-f\left(\gamma_{t}\right)\right| \rightarrow 0$ as $s \rightarrow t$ by continuity, for every $\gamma \in \Gamma(\mathrm{X})$ 
and $t \in[0,1]$. Hence we obtain $(2.11)$ as a consequence of the dominated convergence theorem. Observe also that

$$
L^{1}(\mathfrak{m}) \ni f \longmapsto f \circ \mathrm{e}_{t} \in L^{1}(\boldsymbol{\pi}) \text { is a linear bounded map, for every } t \in[0,1] .
$$

Indeed, $\int\left|f \circ \mathrm{e}_{t}\right| \mathrm{d} \boldsymbol{\pi} \leq \mathrm{C}(\boldsymbol{\pi}) \int|f| \mathrm{d} \mathfrak{m}$ is satisfied for every $f \in L^{1}(\mathfrak{m})$. Now fix $f \in L^{1}(\mathfrak{m})$. Choose a sequence $\left(f_{n}\right)_{n} \subseteq C_{b}(\mathrm{X}) \cap L^{1}(\mathfrak{m})$ that converges to $f$ with respect to the $L^{1}$-norm. Given $t \in[0,1]$ and $n \in \mathbb{N}$, we have that (2.11) and (2.12) yield

$$
\begin{aligned}
\varlimsup_{s \rightarrow t} \int\left|f \circ \mathrm{e}_{s}-f \circ \mathrm{e}_{t}\right| \mathrm{d} \boldsymbol{\pi} & \leq 2 \mathrm{C}(\boldsymbol{\pi})\left\|f-f_{n}\right\|_{L^{1}(\mathfrak{m})}+\varlimsup_{s \rightarrow t} \int\left|f_{n} \circ \mathrm{e}_{s}-f_{n} \circ \mathrm{e}_{t}\right| \mathrm{d} \boldsymbol{\pi} \\
& =2 \mathrm{C}(\boldsymbol{\pi})\left\|f-f_{n}\right\|_{L^{1}(\mathfrak{m})} .
\end{aligned}
$$

By letting $n \rightarrow \infty$ in (2.13), we finally conclude that $\int\left|f \circ \mathrm{e}_{s}-f \circ \mathrm{e}_{t}\right| \mathrm{d} \boldsymbol{\pi} \rightarrow 0$ as $s \rightarrow t$, which proves (2.10). The last statement is obvious.

Under further assumptions on $(\mathrm{X}, \mathrm{d}, \mathfrak{m})$, we have at disposal also a notion of 'speed' $\boldsymbol{\pi}_{t}^{\prime}$ of the test plan $\boldsymbol{\pi}$ at time $t$, as described in the following result. For the proof of such fact, we refer to Theorem 2.3.18 in [15] or Theorem/Definition 1.32 in $[14]$.

Theorem 2.12 (Speed of a test plan). Let (X, d, m) be a metric measure space such that $L^{2}(T \mathrm{X})$ is separable. Let $\boldsymbol{\pi}$ be a test plan on $\mathrm{X}$. Then there exists a unique (up to $\mathcal{L}_{1}$-a.e. equality) family $\boldsymbol{\pi}_{t}^{\prime} \in \mathrm{e}_{t}^{*} L^{2}(T \mathrm{X})$ such that

$$
\exists L^{1}(\boldsymbol{\pi})-\lim _{h \rightarrow 0} \frac{f \circ \mathrm{e}_{t+h}-f \circ \mathrm{e}_{t}}{h}=\left(\mathrm{e}_{t}^{*} \mathrm{~d} f\right)\left(\boldsymbol{\pi}_{t}^{\prime}\right) \quad \text { for } \mathcal{L}^{1} \text {-a.e. } t \in[0,1],
$$

for every $f \in W^{1,2}(\mathrm{X})$. Moreover, the function $(\gamma, t) \mapsto\left|\boldsymbol{\pi}_{t}^{\prime}\right|(\gamma)$ is (the equivalence class of ) a Borel map such that for $\mathcal{L}^{1}$-a.e. $t \in[0,1]$ it holds that

$$
\left|\boldsymbol{\pi}_{t}^{\prime}\right|(\gamma)=\left|\dot{\gamma}_{t}\right| \quad \text { for } \boldsymbol{\pi} \text {-a.e. } \gamma \in A C([0,1], \mathrm{X}) .
$$

Proposition 2.13. Let $(\mathrm{X}, \mathrm{d}, \mathfrak{m})$ be a metric measure space such that $L^{2}(T \mathrm{X})$ is separable, let $\boldsymbol{\pi}$ be a test plan on $\mathrm{X}$ and $f \in W^{1,2}(\mathrm{X})$. Then the a.e. defined map $[0,1] \ni t \mapsto \mathrm{e}_{t}^{*} \mathrm{~d} f\left(\boldsymbol{\pi}_{t}^{\prime}\right) \in L^{1}(\boldsymbol{\pi})$ is a.e. equal to a Borel map.

Proof. For every $h \in(0,1)$ the map $[0,1-h] \ni t \mapsto\left(f \circ \mathrm{e}_{t+h}-f \circ \mathrm{e}_{t}\right) / h \in L^{1}(\boldsymbol{\pi})$ is continuous. Thus by classical arguments the set of $t$ 's for which the limit as $h \rightarrow 0$ exists is Borel and the limit function, set, say, to 0 when the limit does not exist, is Borel.

In this paper we shall work only on RCD spaces, which in particular are so that $W^{1,2}(\mathrm{X})$ is reflexive. In turn this implies, by the arguments in [1], that the tangent module is separable, so that the assumptions of Theorem 2.12 and Proposition 2.13 above are fulfilled.

In the sequel, we will mainly focus our attention on those test plans $\pi$ that are concentrated on an equiLipschitz family of curves. As illustrated in the next definition, we will refer to them as 'Lipschitz test plans'. 
Definition 2.14 (Lipschitz test plan). Let $(\mathrm{X}, \mathrm{d}, \mathfrak{m})$ be a metric measure space such that $L^{2}(T \mathrm{X})$ is separable. Then a test plan $\pi$ on $\mathrm{X}$ is said to be a Lipschitz test plan provided there exists a constant $\mathrm{L} \geq 0$ such that

$$
\left|\boldsymbol{\pi}_{t}^{\prime}\right| \leq \mathrm{L} \text { holds } \boldsymbol{\pi} \text {-a.e. in } \Gamma(\mathrm{X}), \quad \text { for } \mathcal{L}^{1} \text {-a.e. } t \in[0,1],
$$

or, equivalently, such that $\boldsymbol{\pi}$ is concentrated on the family of all the L-Lipschitz curves in $X$. The smallest constant $L \geq 0$ for which (2.16) is satisfied will be denoted by $L(\boldsymbol{\pi})$.

Whenever the test plan $\boldsymbol{\pi}$ is Lipschitz, we have $\left(\mathrm{e}_{t}^{*} \mathrm{~d} f\right)\left(\boldsymbol{\pi}_{t}^{\prime}\right) \in L^{2}(\boldsymbol{\pi})$ for every $f \in W^{1,2}(\mathrm{X})$. One is then led to wonder whether in this case the limit in $(2.14)$ takes place not only in $L^{1}(\boldsymbol{\pi})$, but also in $L^{2}(\boldsymbol{\pi})$. The answer is affirmative, as shown in the following simple result.

Proposition 2.15. Let $(\mathrm{X}, \mathrm{d}, \mathfrak{m})$ be a metric measure space such that $L^{2}(T \mathrm{X})$ is separable. Let $\boldsymbol{\pi}$ be a Lipschitz test plan on $\mathrm{X}$. Let $f \in W^{1,2}(\mathrm{X})$. Then the mapping $t \mapsto f \circ \mathrm{e}_{t} \in L^{2}(\boldsymbol{\pi})$ is Lipschitz and

$$
L^{2}(\boldsymbol{\pi})-\frac{\mathrm{d}}{\mathrm{d} t}\left(f \circ \mathrm{e}_{t}\right)=\left(\mathrm{e}_{t}^{*} \mathrm{~d} f\right)\left(\boldsymbol{\pi}_{t}^{\prime}\right) \quad \text { for } \mathcal{L}^{1} \text {-a.e. } t \in[0,1],
$$

where $L^{2}(\boldsymbol{\pi})-\frac{\mathrm{d}}{\mathrm{d} t}$ stands for the strong derivative (with respect to $t$ ) in the Banach space $L^{2}(\boldsymbol{\pi})$.

Proof. Given any $t, s \in[0,1]$ with $s<t$, one has that

$$
\left\|f \circ \mathrm{e}_{t}-f \circ \mathrm{e}_{s}\right\|_{L^{2}(\boldsymbol{\pi})}^{2}=\int\left|f\left(\gamma_{t}\right)-f\left(\gamma_{s}\right)\right|^{2} \mathrm{~d} \boldsymbol{\pi}(\gamma)
$$

(by definition of Sobolev functions) $\leq \int\left(\int_{s}^{t}|D f|\left(\gamma_{r}\right)\left|\dot{\gamma}_{r}\right| \mathrm{d} r\right)^{2} \mathrm{~d} \boldsymbol{\pi}(\gamma)$

$$
\begin{aligned}
\text { (by Hölder's inequality) } & \leq(t-s) \mathrm{L}(\boldsymbol{\pi})^{2} \iint_{s}^{t}|D f|^{2}\left(\gamma_{r}\right) \mathrm{d} r \mathrm{~d} \boldsymbol{\pi}(\gamma) \\
& \leq \mathrm{C}(\boldsymbol{\pi}) \mathrm{L}(\boldsymbol{\pi})^{2}\|f\|_{W^{1,2}(\mathrm{X})}^{2}(t-s)^{2}
\end{aligned}
$$

which shows that $t \mapsto f \circ \mathrm{e}_{t} \in L^{2}(\boldsymbol{\pi})$ is a Lipschitz map. In particular, it is differentiable at almost every $t \in[0,1]$ by Theorem 2.4 , so that $(2.17)$ follows from (2.14).

\section{Introduction of appropriate functional spaces}

Throughout all this chapter, $(\mathbf{X}, \mathrm{d}, \mathfrak{m})$ is a given $\operatorname{RCD}(K, \infty)$ space and $\boldsymbol{\pi}$ a test plan on it. Recall that the $\operatorname{space} \operatorname{TestF}(\mathrm{X})$ of test functions on $\mathrm{X}$ is defined as

$$
\operatorname{TestF}(\mathrm{X}):=\left\{f \in L^{\infty} \cap \operatorname{LIP} \cap W^{1,2}(\mathrm{X}) \cap D(\Delta): \Delta f \in W^{1,2}(\mathrm{X})\right\}
$$

and that the space of test vector fields on $\mathrm{X}$ is defined as

$$
\operatorname{TestV}(\mathrm{X}):=\left\{\sum_{i=1}^{n} f_{i} \nabla g_{i}: n \in \mathbb{N}^{+}, f_{i}, g_{i} \in \operatorname{TestF}(\mathrm{X}) \text { for every } i\right\} \subset L^{2}(T \mathrm{X}) \text {. }
$$




\subsection{Test vector fields along $\pi$}

We define the space of vector fields along $\boldsymbol{\pi}$ as:

$$
\mathrm{VF}(\boldsymbol{\pi}):=\prod_{t \in[0,1]} \mathrm{e}_{t}^{*} L^{2}(T \mathrm{X})
$$

Thus $\operatorname{VF}(\boldsymbol{\pi})$ is the collection of maps assigning to each $t \in[0,1]$ an element of $\mathrm{e}_{t}^{*} L^{2}(T \mathrm{X})$; it is a vector space w.r.t. pointwise operation.

To each $V \in \operatorname{VF}(\boldsymbol{\pi})$ we associate the function $\llbracket V \rrbracket:[0,1] \rightarrow[0,+\infty)$, defined by

$$
\llbracket V \rrbracket_{t}:=\left\|V_{t}\right\|_{\mathrm{e}_{t}^{*} L^{2}(T \mathrm{X})} \quad \text { for every } t \in[0,1] .
$$

The subspace TestVF $(\boldsymbol{\pi}) \subset \operatorname{VF}(\boldsymbol{\pi})$ of test vector fields along $\boldsymbol{\pi}$ is defined as:

$\operatorname{TestVF}(\boldsymbol{\pi}):=\left\{\begin{array}{l|l}t \mapsto \sum_{i=1}^{n} \varphi_{i}(t) \chi_{A_{i}} \mathrm{e}_{t}^{*} v_{i} & \begin{array}{l}n \in \mathbb{N}^{+}, A_{i} \in \mathscr{B}(\Gamma(\mathrm{X})), \varphi_{i} \in \operatorname{LIP}([0,1]), \\ \text { and } v_{i} \in \operatorname{TestV}(\mathrm{X}) \text { for every } i=1, \ldots, n\end{array}\end{array}\right\}$

Since $\operatorname{TestV}(\mathrm{X}) \subseteq L^{\infty}(T \mathrm{X})$ we see that for any $V \in \operatorname{TestVF}(\boldsymbol{\pi})$ the function $(\gamma, t) \mapsto\left|V_{t}\right|(\gamma)$ belongs to $L^{\infty}\left(\mathcal{L}_{1} \times \boldsymbol{\pi}\right)$.

Proposition 3.1 (Continuity of the test vector fields along $\boldsymbol{\pi}$ ). For any $V, W \in$ $\operatorname{TestVF}(\boldsymbol{\pi})$ we have that

$$
[0,1] \ni t \rightarrow\left\langle V_{t}, W_{t}\right\rangle \in L^{1}(\boldsymbol{\pi}) \quad \text { is continuous. }
$$

In particular, the function $\llbracket V \rrbracket:[0,1] \rightarrow[0,+\infty)$ is continuous for every $V \in$ $\operatorname{Test} \Gamma(\mathrm{X})$.

Proof. By linearity, it is clear that it is sufficient to prove the claim for $V, W$ of the form $V=\chi_{A} \mathrm{e}_{t}^{*} v, W=\chi_{B} \mathrm{e}_{t}^{*} w$ for $v, w \in \operatorname{TestV}(\mathrm{X})$. In this case the claim (3.1) is a direct consequence of

$$
\left\langle V_{t}, W_{t}\right\rangle=\chi_{A \cap B}\langle v, w\rangle \circ \mathrm{e}_{t}
$$

and Theorem 2.11. The last statement follows by choosing $W=V$.

We now define two norms on $\operatorname{TestVF}(\boldsymbol{\pi})$ :

$$
\|V\|_{\mathscr{L}^{2}(\boldsymbol{\pi})}^{2}:=\int_{0}^{1} \llbracket V \rrbracket_{t}^{2} \mathrm{~d} t \quad \text { and } \quad\|V\|_{\mathscr{C}(\boldsymbol{\pi})}:=\max _{t \in[0,1]} \llbracket V \rrbracket_{t} .
$$

Notice that Proposition 3.1 ensures that $t \mapsto \llbracket V \rrbracket_{t}$ is Borel, hence $\|\cdot\|_{\mathscr{L}^{2}(\boldsymbol{\pi})}$ is well defined; also, routine computations show that $\|\cdot\|_{\mathscr{L}^{2}(\boldsymbol{\pi})},\|\cdot\|_{\mathscr{C}(\boldsymbol{\pi})}$ are norms on $\operatorname{TestVF}(\boldsymbol{\pi})$ with $\|\cdot\|_{\mathscr{L}^{2}(\boldsymbol{\pi})} \leq\|\cdot\|_{\mathscr{C}(\boldsymbol{\pi})}$.

We now want to show that $\left(\operatorname{TestVF}(\boldsymbol{\pi}),\|\cdot\|_{\mathscr{C}(\boldsymbol{\pi})}\right)$ is separable by exhibiting a countable dense subset. To this aim, we first choose three countable families $\mathcal{F}_{1} \subseteq\{$ open sets of $\Gamma(\mathrm{X})\}, \mathcal{F}_{2} \subseteq \operatorname{LIP}([0,1])$ and $\mathcal{F}_{3} \subseteq \operatorname{TestF}(\mathrm{X})$ such that

given $A \subseteq \Gamma(\mathrm{X})$ Borel and $\varepsilon>0$, there exists $U \in \mathcal{F}_{1}$ with $\boldsymbol{\pi}(A \Delta U)<\varepsilon$, $\mathcal{F}_{2}$ is dense in $C([0,1])$ and stable by product and $\mathbb{Q}$-linear combinations, $\mathcal{F}_{3}$ is a $\mathbb{Q}$-vector space of functions in $W^{1,2}(\mathrm{X})$ whose gradients generate $L^{2}(T \mathrm{X})$. 
We proceed in the following way:

$\mathcal{F}_{1}$ : Since $\Gamma(X)$ is separable, there exists a countable family $\widetilde{\mathcal{F}}_{1}$ of open subsets of $\Gamma(\mathrm{X})$ that is a neighbourhood basis for each point $\gamma \in \Gamma(\mathrm{X})$. Let us denote by $\mathcal{F}_{1}$ the set of finite unions of elements of $\widetilde{\mathcal{F}}_{1}$, so that $\mathcal{F}_{1}$ is countable. Fix $A \in \mathscr{B}(\Gamma(\mathrm{X}))$ and $\varepsilon>0$. The measure $\pi$ is regular, since $\left(\Gamma(\mathrm{X}), \mathrm{d}_{\Gamma(\mathrm{X})}\right)$ is complete and separable. By inner regularity of $\boldsymbol{\pi}$, there exists a compact subset $K \subseteq A$ such that $\pi(A \backslash K)<\varepsilon / 2$. By outer regularity of $\boldsymbol{\pi}$, there exists $V \subseteq \Gamma(\mathrm{X})$ open such that $K \subseteq V$ and $\boldsymbol{\pi}(V \backslash K)<\varepsilon / 2$. We can then associate to any $\gamma \in K$ a set $U_{\gamma} \in \widetilde{\mathcal{F}}_{1}$ such that $\gamma \in U_{\gamma} \subseteq V$. By compactness of $K$, one has $K \subseteq U_{\gamma_{1}} \cup \ldots \cup U_{\gamma_{n}} \subseteq V$ for some finite choice $\gamma_{1}, \ldots, \gamma_{n} \in K$. Let us call $U:=U_{\gamma_{1}} \cup \ldots \cup U_{\gamma_{n}} \in \mathcal{F}_{1}$. We thus have that

$$
\boldsymbol{\pi}(A \Delta U)=\boldsymbol{\pi}(A \backslash U)+\boldsymbol{\pi}(U \backslash A) \leq \boldsymbol{\pi}(A \backslash K)+\boldsymbol{\pi}(V \backslash K)<\varepsilon .
$$

$\mathcal{F}_{2}$ : By the separability of $C([0,1])$, such $\mathcal{F}_{2}$ exists.

$\mathcal{F}_{3}$ : Since the space $(\mathrm{X}, \mathrm{d}, \mathfrak{m})$ is infinitesimally Hilbertian, we have that $W^{1,2}(\mathrm{X})$ is reflexive and therefore, by [1], separable. Thus let $\mathcal{F}_{3}$ be any countable dense $\mathbb{Q}$-vector subspace of $W^{1,2}(\mathrm{X})$ and notice that since gradients of functions in $W^{1,2}(\mathrm{X})$ generate $L^{2}(T \mathrm{X})$, the same holds for functions in $\mathcal{F}_{3}$.

We now define the class of test vector fields $\operatorname{TestVF}_{\mathbb{N}}(\boldsymbol{\pi})$ as

$$
\operatorname{TestVF}_{\mathbb{N}}(\boldsymbol{\pi}):=\left\{\begin{array}{l|l}
t \mapsto \sum_{i=1}^{n} \psi_{i}(t) \chi_{U_{i}} \mathrm{e}_{t}^{*} \nabla f_{i} & \begin{array}{l}
n \in \mathbb{N}^{+} \text {and } U_{i} \in \mathcal{F}_{1}, \psi_{i} \in \mathcal{F}_{2}, \\
f_{i} \in \mathcal{F}_{3} \text { for every } i=1, \ldots, n
\end{array}
\end{array}\right\} .
$$

Clearly, $\operatorname{TestVF}_{\mathbb{N}}(\boldsymbol{\pi})$ is a countable subset of $\operatorname{TestVF}(\boldsymbol{\pi})$. Also, notice that the inequalities

$$
\begin{aligned}
& \llbracket \chi_{A} \mathrm{e}_{t}^{*} v-\chi_{A} \mathrm{e}_{t}^{*} w \rrbracket_{t} \leq \sqrt{\mathrm{C}(\boldsymbol{\pi})}\|v-w\|_{L^{2}(T \mathrm{X})}, \\
& \llbracket \chi_{A} \mathrm{e}_{t}^{*} v-\chi_{U} \mathrm{e}_{t}^{*} v \rrbracket_{t} \leq \sqrt{\boldsymbol{\pi}(A \Delta U)}\|v\|_{L^{\infty}(T \mathrm{X})},
\end{aligned}
$$

valid for any $t \in[0,1], A, U \subset \Gamma(\mathrm{X})$ Borel and $v, w \in L^{2}(T \mathrm{X})$, and the very definition of pullback module, show that

(3.2) for any $t \in[0,1]$ the set $\left\{W_{t}: W \in \operatorname{TestVF}_{\mathbb{N}}(\boldsymbol{\pi})\right\}$ is dense in $\mathrm{e}_{t}^{*} L^{2}(T \mathrm{X})$.

Lemma 3.2 (Separability of $\operatorname{TestVF}(\boldsymbol{\pi})$ ). The family $\operatorname{TestVF}_{\mathbb{N}}(\boldsymbol{\pi})$ is dense in $\operatorname{TestVF}(\boldsymbol{\pi})$ with respect to the norm $\|\cdot\|_{\mathscr{C}(\boldsymbol{\pi})}\left(\right.$ and thus also w.r.t. $\|\cdot\|_{\mathscr{L}^{2}(\boldsymbol{\pi})}$ ).

Proof. Let $V \in \operatorname{TestVF}(\boldsymbol{\pi})$ be arbitrary, $\varepsilon>0$ and $t_{0} \in[0,1]$. By (3.2) there is $W \in \operatorname{TestVF}_{\mathbb{N}}(\boldsymbol{\pi})$ such that $\llbracket V-W \rrbracket_{t_{0}}<\varepsilon$. Since $t \mapsto \llbracket V-W \rrbracket_{t}^{2}=\llbracket V \rrbracket_{t}^{2}+$ $\llbracket W \rrbracket_{t}^{2}-2 \int\left\langle V_{t}, W_{t}\right\rangle \mathrm{d} \boldsymbol{\pi}$ is continuous, we see that $\llbracket V-W \rrbracket_{t}<\varepsilon$ for every $t$ in a neighbourhood of $t_{0}$. By compactness of $[0,1]$, we can then find a finite number of open intervals $I_{1}, \ldots, I_{n}$ covering $[0,1]$ and elements $W_{1}, \ldots, W_{n} \in \operatorname{TestVF}_{\mathbb{N}}(\boldsymbol{\pi})$ such that

$$
\llbracket V-W_{i} \rrbracket_{t}<\varepsilon, \quad \forall t \in I_{i} \cap[0,1], i=1, \ldots, n .
$$


By multiplying $W_{i}$ by an appropriate function in $\mathcal{F}_{2}$ we can also assume that

$$
\llbracket W_{i} \rrbracket_{t}<\|V\|_{\mathscr{C}(\boldsymbol{\pi})}+2 \varepsilon, \quad \forall t \in[0,1]
$$

Now let $\left(\phi_{i}\right)$ be a Lipschitz partition of the unity subordinate to the cover made with the $I_{i}$ 's and for any $i$ let $\psi_{i} \in \mathcal{F}_{2}$ be such that $\left|\phi_{i}(t)-\psi_{i}(t)\right|<\varepsilon$ for every $t \in[0,1]$. Then we have $W_{t}:=\sum_{i} \psi_{i}(t) W_{i, t} \in \operatorname{TestVF}_{\mathbb{N}}(\boldsymbol{\pi})$ and

$$
\llbracket V-W \rrbracket_{t} \leq \llbracket V-\sum_{i} \phi_{i}(t) W_{i} \rrbracket_{t}+\llbracket \sum_{i}\left(\psi_{i}(t)-\phi_{i}(t)\right) W_{i} \rrbracket_{t} \stackrel{(3.3),(3.4)}{\leq} \varepsilon+\varepsilon\left(\|V\|_{\mathscr{C}(\boldsymbol{\pi})}+2 \varepsilon\right)
$$

for any $t \in[0,1]$. The conclusion follows by the arbitrariness of $\varepsilon>0$.

\subsection{The space $\mathscr{L}^{2}(\pi)$}

We start defining the class of Borel vector fields along $\boldsymbol{\pi}$.

Definition 3.3 (Borel vector fields along $\boldsymbol{\pi}$ ). We say that $V \in \operatorname{VF}(\boldsymbol{\pi})$ is Borel provided

$$
[0,1] \ni t \longmapsto \int\left\langle V_{t}, W_{t}\right\rangle \mathrm{d} \boldsymbol{\pi} \quad \text { is a Borel function, }
$$

for every $W \in \operatorname{TestVF}_{\mathbb{N}}(\boldsymbol{\pi})$.

Notice that thanks to Lemma 3.2 this notion would be unaltered if we require (3.5) to hold for any $W \in \operatorname{TestVF}(\boldsymbol{\pi})$. Also, Proposition 3.1 ensures that test vector fields are Borel. We have the following basic result.

Proposition 3.4. Let $V \in \operatorname{VF}(\boldsymbol{\pi})$ be Borel. Then the map $\llbracket V \rrbracket:[0,1] \rightarrow[0, \infty)$ is Borel.

Proof. From (3.2) we deduce that

$$
\llbracket V \rrbracket_{t}^{2}=\sup _{W \in \operatorname{Test} \Gamma_{\mathbb{N}}(\mathrm{X})}\left(2 \int\left\langle V_{t}, W_{t}\right\rangle \mathrm{d} \boldsymbol{\pi}-\llbracket W \rrbracket_{t}^{2}\right) \quad \text { for every } t \in[0,1],
$$

and the thesis follows.

We can now define the space $\mathscr{L}^{2}(\boldsymbol{\pi})$.

Definition 3.5 (The space $\mathscr{L}^{2}(\boldsymbol{\pi})$ ). We define $\mathscr{L}^{2}(\boldsymbol{\pi})$ as the space of all Borel vector fields $V \in \operatorname{VF}(\boldsymbol{\pi})$ such that

$$
\|V\|_{\mathscr{L}^{2}(\boldsymbol{\pi})}^{2}:=\int_{0}^{1} \llbracket V \rrbracket_{t}^{2} \mathrm{~d} t=\int_{0}^{1} \int\left|V_{t}\right|^{2} \mathrm{~d} \boldsymbol{\pi} \mathrm{d} t<+\infty
$$

where we identify $V, \tilde{V} \in \operatorname{VF}(\boldsymbol{\pi})$ if $V_{t}=\tilde{V}_{t}$ for a.e. $t \in[0,1]$.

Clearly $\left(\mathscr{L}^{2}(\boldsymbol{\pi}),\|\cdot\|_{\mathscr{L}^{2}(\boldsymbol{\pi})}\right)$ is a normed space, wherein $\operatorname{TestVF}(\boldsymbol{\pi})$ is embedded. The following result can be obtained by adapting the classical arguments concerning the standard $L^{2}$ spaces. For completeness we report its proof, the context being different from the usual one. 
Proposition 3.6 (Basic properties of $\mathscr{L}^{2}(\boldsymbol{\pi})$ ). The space $\mathscr{L}^{2}(\boldsymbol{\pi})$ is a Hilbert space, and if $V_{n} \rightarrow V$ in $\mathscr{L}^{2}(\boldsymbol{\pi})$ then there is a subsequence such that $V_{n, t} \rightarrow V_{t}$ in $\mathrm{e}_{t}^{*} L^{2}(T \mathrm{X})$ for a.e. $t \in[0,1]$.

Proof. It is clear that the $\mathscr{L}^{2}(\boldsymbol{\pi})$ norm comes from the scalar product

$$
\langle V, W\rangle_{\mathscr{L}^{2}(\boldsymbol{\pi})}:=\int_{0}^{1} \int\left\langle V_{t}, W_{t}\right\rangle \mathrm{d} \boldsymbol{\pi} \mathrm{d} t .
$$

To conclude the proof we shall show that if $\left(V_{n}\right)$ is a sequence of Borel vector fields in $\mathscr{L}^{2}(\boldsymbol{\pi})$ such that $\sum_{n}\left\|V_{n+1}-V_{n}\right\|_{\mathscr{L}^{2}(\boldsymbol{\pi})}<\infty$, then such sequence has a limit $V \in \mathscr{L}^{2}(\boldsymbol{\pi})$ and for a.e. $t$ it holds $V_{n, t} \rightarrow V_{t}$ in $\mathrm{e}_{t}^{*} L^{2}(T \mathrm{X})$.

Define the Borel function $g:[0,1] \rightarrow[0,+\infty]$ as $g:=\sum_{n} \llbracket V_{n+1}-V_{n} \rrbracket$ and notice that since, for all $N \in \mathbb{N}$,

$$
\left\|\sum_{n=1}^{N} \llbracket V_{n+1}-V_{n} \rrbracket\right\|_{L^{2}(0,1)} \leq \sum_{n=1}^{N}\left\|V_{n+1}-V_{n}\right\|_{\mathscr{L}^{2}(\boldsymbol{\pi})} \leq \sum_{n=1}^{\infty}\left\|V_{n+1}-V_{n}\right\|_{\mathscr{L}^{2}(\boldsymbol{\pi})}<\infty,
$$

we have that $g \in L^{2}(0,1)$. Let $N:=\{t: g(t)=+\infty\}$ and notice that for every $t \in[0,1] \backslash N$ we have

$$
\sum_{n=1}^{\infty}\left\|V_{n+1, t}-V_{n, t}\right\|_{\mathrm{e}_{t}^{*} L^{2}(T \mathrm{X})}=\sum_{n=1}^{\infty} \llbracket V_{n+1}-V_{n} \rrbracket_{t}=g(t)<\infty,
$$

proving that $\left(V_{n, t}\right)$ is a Cauchy sequence in $\mathrm{e}_{t}^{*} L^{2}(T \mathrm{X})$. Then define

$$
V_{t}:= \begin{cases}\lim _{n} V_{n, t} \in \mathrm{e}_{t}^{*} L^{2}(T \mathrm{X}), & \text { if } t \in[0,1] \backslash N \\ 0 \in \mathrm{e}_{t}^{*} L^{2}(T \mathrm{X}), & \text { if } t \in N\end{cases}
$$

Notice that for every $W \in \operatorname{TestVF}(\boldsymbol{\pi})$ we have $\int\left\langle V_{t}, W_{t}\right\rangle \mathrm{d} \boldsymbol{\pi}=\lim _{n} \int\left\langle V_{n, t}, W_{t}\right\rangle \mathrm{d} \boldsymbol{\pi}$ for all $t \in[0,1] \backslash N$, hence the map $[0,1] \ni t \mapsto \int\left\langle V_{t}, W_{t}\right\rangle \mathrm{d} \boldsymbol{\pi}$ is Borel and, by arbitrariness of $W$, this shows that $V$ is Borel. Since trivially we have $\llbracket V \rrbracket_{t} \leq$ $\llbracket V_{1} \rrbracket_{t}+\sum_{n=1}^{\infty} \llbracket V_{n+1}-V_{n} \rrbracket_{t}$, by (3.6) we see that $V \in \mathscr{L}^{2}(\boldsymbol{\pi})$. Now to check that $V_{n} \rightarrow V$ in $\mathscr{L}^{2}(\boldsymbol{\pi})$ notice that, again by (3.6), the sequence $\llbracket V-V_{n} \rrbracket_{t}$ is dominated in $L^{2}(0,1)$ and that for every $t \in[0,1] \backslash N$ we have

$$
\lim _{n \rightarrow \infty} \llbracket V-V_{n} \rrbracket_{t} \leq \lim _{n \rightarrow \infty} \lim _{m \rightarrow \infty} \sum_{i=n}^{m} \llbracket V_{i+1}-V_{i} \rrbracket_{t} \stackrel{(3.6)}{=} 0,
$$

so that the conclusion follows by the dominated convergence theorem.

Proposition 3.7 (Density of $\operatorname{TestVF}_{\mathbb{N}}(\boldsymbol{\pi})$ in $\mathscr{L}^{2}(\boldsymbol{\pi})$ ). The space $\operatorname{TestVF}_{\mathbb{N}}(\boldsymbol{\pi})$ is dense in $\mathscr{L}^{2}(\boldsymbol{\pi})$. In particular, $\mathscr{L}^{2}(\boldsymbol{\pi})$ is separable.

Proof. Let $\left(Z_{k}\right)$ be an enumeration of the elements in $\operatorname{TestVF}_{\mathbb{N}}(\boldsymbol{\pi})$, pick a Borel vector field $V \in \mathscr{L}^{2}(\boldsymbol{\pi})$ and choose $\varepsilon>0$. Then for every $k \in \mathbb{N}$ let $\widetilde{G}_{k}:=\{t \in$ $\left.[0,1]: \llbracket V-Z_{k} \rrbracket_{t}<\varepsilon\right\}$ and put $G_{1}:=\tilde{G}_{1}$ and $G_{k}:=\widetilde{G}_{k} \backslash\left(\widetilde{G}_{1} \cup \ldots \cup \widetilde{G}_{k-1}\right)$ for $k>1$. Then (3.2) grants that $\left(G_{k}\right)_{k \geq 1}$ is a Borel partition of $[0,1]$. 
Now for $m \in \mathbb{N} \cup\{\infty\}$ define $W_{m} \in \mathscr{L}^{2}(\boldsymbol{\pi})$ as $W_{m, t}:=\sum_{k=1}^{m} \chi_{G_{k}}(t) Z_{k, t}$. Observe that $\left\|W_{\infty}-V\right\|_{\mathscr{L}^{2}(\boldsymbol{\pi})}<\varepsilon$ by definition of $G_{k}$. Moreover, for each $m \geq 1$ one has that

$$
\left\|W_{m}-W_{\infty}\right\|_{\mathscr{L}^{2}(\boldsymbol{\pi})}^{2}=\sum_{k=m+1}^{\infty} \int_{G_{k}} \llbracket Z_{k} \rrbracket_{t}^{2} \mathrm{~d} t \leq \int_{\bigcup_{k>m} G_{k}} 2\left(\llbracket V \rrbracket_{t}^{2}+\varepsilon^{2}\right) \mathrm{d} t
$$

so that accordingly $\lim _{m \rightarrow \infty}\left\|W_{m}-W_{\infty}\right\|_{\mathscr{L}^{2}(\boldsymbol{\pi})}=0$ by the dominated convergence theorem.

Hence to conclude it is sufficient to show that each $W_{m}$ belongs to the $\mathscr{L}^{2}(\boldsymbol{\pi})$ closure of $\operatorname{TestVF}_{\mathbb{N}}(\boldsymbol{\pi})$ and in turn this will follow if we prove that for $Z \in$ $\operatorname{TestVF}_{\mathbb{N}}(\boldsymbol{\pi})$ and $G \subset[0,1]$ Borel the vector field $\chi_{G} Z$ belongs to the $\mathscr{L}^{2}(\boldsymbol{\pi})$-closure of $\operatorname{TestVF}_{\mathbb{N}}(\boldsymbol{\pi})$. To see this, simply let $\left(\varphi_{n}\right) \subset \operatorname{LIP}([0,1])$ be uniformly bounded and a.e. converging to $\chi_{G}$, notice that $\varphi_{n} Z \in \operatorname{TestVF}(\boldsymbol{\pi})$ and that an application of the dominate convergence theorem shows that $\varphi_{n} Z \rightarrow \chi_{G} Z$ in $\mathscr{L}^{2}(\pi)$.

Now consider the speed $\boldsymbol{\pi}_{t}^{\prime}$, associated to any test plan $\pi$ by Theorem 2.12 .

Proposition 3.8. The (equivalence class up to a.e. equality of the) map $t \mapsto \boldsymbol{\pi}_{t}^{\prime}$ is an element of the space $\mathscr{L}^{2}(\boldsymbol{\pi})$.

Proof. We have $\boldsymbol{\pi}_{t}^{\prime} \in \mathrm{e}_{t}^{*} L^{2}(T \mathrm{X})$ for a.e. $t \in[0,1]$ and

$$
\int_{0}^{1} \int\left|\boldsymbol{\pi}_{t}^{\prime}\right|^{2} \mathrm{~d} \boldsymbol{\pi} \mathrm{d} t \stackrel{(2.15)}{=} \int_{0}^{1} \int\left|\dot{\gamma}_{t}\right|^{2} \mathrm{~d} \boldsymbol{\pi}(\gamma) \mathrm{d} t<+\infty
$$

by the very definition of test plan. Hence we need only to show that $t \mapsto \boldsymbol{\pi}_{t}^{\prime}$ has a Borel representative in the sense of Definition 3.3.

Notice that for any $f \in W^{1,2}(\mathrm{X})$, by Proposition 2.13 we have that the map $t \mapsto$ $\left(\mathrm{e}_{t}^{*} \mathrm{~d} f\right)\left(\boldsymbol{\pi}_{t}^{\prime}\right)$ has a Borel representative. Hence the same holds for $t \mapsto \psi(t) \chi_{U}\left\langle\mathrm{e}_{t}^{*} \nabla f\right.$, $\left.\boldsymbol{\pi}_{t}^{\prime}\right\rangle$ for every $\psi \in \operatorname{LIP}([0,1])$ and $U \subset \Gamma(\mathrm{X})$ Borel. Therefore there exists a Borel negligible set $N \subset[0,1]$ such that for every $V \in \operatorname{TestVF}_{\mathbb{N}}(\boldsymbol{\pi})$ the map $t \mapsto \int\left\langle V_{t}, \boldsymbol{\pi}_{t}^{\prime}\right\rangle \mathrm{d} \boldsymbol{\pi}$, set to 0 on $N$, is Borel. This is sufficient to conclude.

We conclude the section by pointing out that $\mathscr{L}^{2}(\boldsymbol{\pi})$ can also be seen as the pullback of $L^{2}(T \mathbf{X})$ via the evaluation map e: $\Gamma(\mathbf{X}) \times[0,1] \rightarrow \mathbf{X}$ defined as e $(\gamma, t):=\gamma_{t}$ (recall also Remark 2.10). To this aim, let us start by defining the following operations:

(i) Given $f \in L^{\infty}\left(\boldsymbol{\pi} \times \mathcal{L}_{1}\right)$ and $V \in \mathscr{L}^{2}(\boldsymbol{\pi})$, we define $f V \in \mathscr{L}^{2}(\boldsymbol{\pi})$ as

$$
(f V)_{t}:=f(\cdot, t) V_{t} \quad \text { for } \mathcal{L}^{1} \text {-a.e. } t \in[0,1] .
$$

(ii) To each $V \in \mathscr{L}^{2}(\boldsymbol{\pi})$ we associate the map $|V| \in L^{2}\left(\boldsymbol{\pi} \times \mathcal{L}_{1}\right)$, defined by

$$
|V|(\gamma, t):=\left|V_{t}\right|(\gamma) \quad \text { for }\left(\boldsymbol{\pi} \times \mathcal{L}_{1}\right) \text {-a.e. }(\gamma, t) \in \Gamma(\mathrm{X}) \times[0,1] .
$$

It is clear that these operations give $\mathscr{L}^{2}(\boldsymbol{\pi})$ the structure of an $L^{2}\left(\boldsymbol{\pi} \times \mathcal{L}_{1}\right)$ normed module. 
We then define the linear continuous operator $\Phi: L^{2}(T \mathrm{X}) \rightarrow \mathscr{L}^{2}(\boldsymbol{\pi})$ by putting

$$
\Phi(v)_{t}:=\mathrm{e}_{t}^{*} v, \quad \text { for } \mathcal{L}_{1} \text {-a.e. } t \in[0,1] .
$$

We then have:

Proposition $3.9\left(\mathscr{L}^{2}(\boldsymbol{\pi})\right.$ as pullback). We have $\left(\mathscr{L}^{2}(\boldsymbol{\pi}), \Phi\right) \sim\left(\mathrm{e}^{*} L^{2}(T \mathrm{X}), \mathrm{e}^{*}\right)$, i.e.:

$$
\begin{aligned}
|\Phi(v)|=|v| \circ \mathrm{e} & \text { holds }\left(\boldsymbol{\pi} \times \mathcal{L}_{1}\right) \text {-a.e., for any } v \in L^{2}(T \mathrm{X}), \\
\left\{\Phi(v): v \in L^{2}(T \mathrm{X})\right\} & \text { generates } \mathscr{L}^{2}(\boldsymbol{\pi}) \text { as a module. }
\end{aligned}
$$

Proof. The first in (3.8) follows by noticing that $|\Phi(v)|(\gamma, t)=\left|\mathrm{e}_{t}^{*} v\right|(\gamma)=(|v| \circ \mathrm{e})(\gamma, t)$ holds for $\left(\boldsymbol{\pi} \times \mathcal{L}_{1}\right)$-a.e. $(\gamma, t)$, the second one stems from the density of Test $\operatorname{VF}(\boldsymbol{\pi})$ in $\mathscr{L}^{2}(\boldsymbol{\pi})$.

Notice that the notion of pullback module $\left(\mathrm{e}^{*} L^{2}(T \mathrm{X}), \mathrm{e}^{*}\right)$ makes no (explicit) reference to the concept of 'test vector field' as we defined it in Section 3.1. Thus this last proposition is also telling that the choice of using these test object to check Borel regularity, which a priori might seem arbitrary, leads in fact to a canonical interpretation of $\mathscr{L}^{2}(\boldsymbol{\pi})$.

Remark $3.10\left(\mathscr{L}^{2}(\boldsymbol{\pi})\right.$ as direct integral). The construction of $\mathscr{L}^{2}(\boldsymbol{\pi})$ can be summarized by saying that such space is the direct integral of the $\mathrm{e}_{t}^{*} L^{2}(T \mathrm{X})$, the space of Borel vector fields being the so-called 'weakly measurable selections' and the set $\operatorname{TestVF}_{\mathbb{N}}(\boldsymbol{\pi})$ being the one used to check measurability.

\subsection{The space $\mathscr{C}(\pi)$}

Here we introduce and briefly study those vector fields in $\operatorname{VF}(\boldsymbol{\pi})$ which are 'continuous in time'. We start with the following definition.

Definition 3.11 (The space $\mathscr{C}(\boldsymbol{\pi})$ ). Let $V \in \operatorname{VF}(\boldsymbol{\pi})$. Then we say that $V$ is a continuous vector field provided

$$
[0,1] \ni t \mapsto \int\left\langle V_{t}, W_{t}\right\rangle \mathrm{d} \boldsymbol{\pi} \quad \text { is continuous }
$$

for every $W \in \operatorname{TestVF}_{\mathbb{N}}(\boldsymbol{\pi})$ and

$$
[0,1] \ni t \mapsto \llbracket V \rrbracket_{t} \quad \text { is continuous. }
$$

We denote the family of all continuous vector fields by $\mathscr{C}(\boldsymbol{\pi})$ and, for every $V \in$ $\mathscr{C}(\boldsymbol{\pi})$, we put

$$
\|V\|_{\mathscr{C}(\boldsymbol{\pi})}:=\max _{t \in[0,1]} \llbracket V \rrbracket_{t} .
$$

Lemma 3.2 ensures that this definition would be unaltered if we require (3.9) to hold for any $W \in \operatorname{TestVF}(\boldsymbol{\pi})$. Also, Proposition 3.1 gives that $\operatorname{TestVF}(\boldsymbol{\pi}) \subset \mathscr{C}(\boldsymbol{\pi})$.

It is not obvious that $\mathscr{C}(\boldsymbol{\pi})$ is a vector space, the problem being in checking that (3.10) holds for linear combinations. This will be a consequence of the density of $\operatorname{TestVF}_{\mathbb{N}}(\boldsymbol{\pi})$ in $\mathscr{C}(\boldsymbol{\pi})$, which is part of the content of the next result. 
Proposition 3.12. $\left(\mathscr{C}(\boldsymbol{\pi}),\|\cdot\|_{\mathscr{C}(\boldsymbol{\pi})}\right)$ is a separable Banach space, with $\operatorname{TestVF}_{\mathbb{N}}(\boldsymbol{\pi})$ being dense.

Proof. Let $V_{1}, V_{2} \in \mathscr{C}(\boldsymbol{\pi})$ and notice that using (3.9), (3.10) and arguing exactly as in the proof of Lemma 3.2 we can find $\left(W_{1, n}\right),\left(W_{2, n}\right) \subset \operatorname{TestVF}_{\mathbb{N}}(\boldsymbol{\pi})$ such that the functions $t \mapsto \llbracket V_{i}-W_{i, n} \rrbracket_{t}$ uniformly converge to 0 as $n \rightarrow \infty, i=1,2$.

Now observe that since $W_{1, n}+W_{2, n} \in \operatorname{TestVF}_{\mathbb{N}}(\boldsymbol{\pi})$ the map $t \mapsto \llbracket W_{1, n}+W_{2, n} \rrbracket_{t}$ is continuous and that for every $t \in[0,1]$ we have

$\left|\llbracket V_{1}+V_{2} \rrbracket_{t}-\llbracket W_{1, n}+W_{2, n} \rrbracket_{t}\right| \leq \llbracket V_{1}-W_{1, n}+V_{2}-W_{2, n} \rrbracket_{t} \leq \llbracket V_{1}-W_{1, n} \rrbracket_{t}+\llbracket V_{2}-W_{2, n} \rrbracket_{t}$.

Hence $t \mapsto \llbracket V_{1}+V_{2} \rrbracket_{t}$ is the uniform limit of continuous functions and thus continuous itself. Since trivially $\mathscr{C}(\boldsymbol{\pi})$ is closed by multiplication by scalars we proved that it is a vector space. That $\|\cdot\|_{\mathscr{C}(\boldsymbol{\pi})}$ is a complete norm on $\mathscr{C}(\boldsymbol{\pi})$ is trivial and the density of $\operatorname{TestVF}_{\mathbb{N}}(\boldsymbol{\pi})$ has already been shown, hence the proof is finished.

A useful consequence of the density of test vector fields is the following strengthening of the continuity property.

Corollary 3.13. Let $V \in \mathscr{C}(\boldsymbol{\pi})$. Then the map $t \mapsto\left|V_{t}\right|^{2} \in L^{1}(\boldsymbol{\pi})$ is continuous.

Proof. For $V \in \operatorname{TestVF}(\boldsymbol{\pi})$ the claim has been proved in Proposition 3.1. Now notice that for $V, W \in \mathscr{C}(\boldsymbol{\pi})$ we have

$\left.\int|| V_{t}\right|^{2}-\left|W_{t}\right|^{2}\left|\mathrm{~d} \boldsymbol{\pi} \leq \int\right| V_{t}+W_{t}|| V_{t}-W_{t} \mid \mathrm{d} \boldsymbol{\pi} \leq\left(\|V\|_{\mathscr{C}(\boldsymbol{\pi})}+\|W\|_{\mathscr{C}(\boldsymbol{\pi})}\right) \llbracket V-W \rrbracket_{t}$

thus showing that if $V_{n} \rightarrow V$ in $\mathscr{C}(\boldsymbol{\pi})$ then $t \mapsto\left|V_{n, t}\right|^{2} \in L^{1}(\boldsymbol{\pi})$ uniformly converge to $t \mapsto\left|V_{t}\right|^{2} \in L^{1}(\boldsymbol{\pi})$. The conclusion then follows from the density of $\operatorname{TestVF}(\boldsymbol{\pi})$ in $\mathscr{C}(\boldsymbol{\pi})$.

\subsection{The spaces $\mathscr{W}^{1,2}(\pi)$ and $\mathscr{H}^{1,2}(\pi)$}

Throughout all this section we shall further assume that the test plan $\pi$ is Lipschitz in the sense of Definition 2.14.

Let us briefly recall the definitions of $W_{C}^{1,2}(T \mathrm{X})$ and $H_{C}^{1,2}(T \mathrm{X})$, with the corresponding notion of covariant derivative. See Section 3 in [15] for a detailed account on this topic.

First of all, we need to recall the notion of Hessian in this framework: given any function $f \in \operatorname{TestF}(\mathbf{X})$, there exists a unique element $\operatorname{Hess}(f) \in L^{2}\left(\left(T^{*}\right)^{\otimes 2} \mathbf{X}\right)$ that satisfies the identity

$$
\begin{aligned}
& 2 \int h \operatorname{Hess}(f)\left(\nabla g_{1}, \nabla g_{2}\right) \mathrm{d} \mathfrak{m} \\
& =-\int\left\langle\nabla f, \nabla g_{1}\right\rangle \operatorname{div}\left(h \nabla g_{2}\right)+\left\langle\nabla f, \nabla g_{2}\right\rangle \operatorname{div}\left(h \nabla g_{1}\right)+\left\langle\nabla f, \nabla\left\langle\nabla g_{1}, \nabla g_{2}\right\rangle\right\rangle \mathrm{d} \mathfrak{m}
\end{aligned}
$$

for every choice of $h, g_{1}, g_{2} \in \operatorname{TestF}(\mathrm{X})$. 
The resulting notion of Hessian is consistent with the usual one in the smooth setting, as it is an integral reformulation of the well-known identity

$$
\begin{aligned}
2 \operatorname{Hess}(f)\left(\nabla g_{1}, \nabla g_{2}\right)= & \left\langle\nabla\left\langle\nabla f, \nabla g_{1}\right\rangle, \nabla g_{2}\right\rangle \\
& +\left\langle\nabla\left\langle\nabla f, \nabla g_{2}\right\rangle, \nabla g_{1}\right\rangle+\left\langle\nabla f, \nabla\left\langle\nabla g_{1}, \nabla g_{2}\right\rangle\right\rangle .
\end{aligned}
$$

With the Hessian at disposal, one can define the covariant derivative of a vector field on $\mathrm{X}$ as follows: we say that $v \in L^{2}(T \mathrm{X})$ belongs to the space $W_{C}^{1,2}(T \mathrm{X})$ provided there exists an element $\nabla v \in L^{2}\left(T^{\otimes 2} \mathrm{X}\right)$ - which is unique and called covariant derivative of $v$-such that

$$
\int h \nabla v:(\nabla f \otimes \nabla g) \mathrm{d} \mathfrak{m}=-\int\langle v, \nabla g\rangle \operatorname{div}(h \nabla f)+h \operatorname{Hess}(g)(v, \nabla f) \mathrm{d} \mathfrak{m}
$$

for every $f, g, h \in \operatorname{TestF}(\mathbf{X})$. Also this notion is consistent with the classical one in the smooth world, as it is based upon the identity $\left\langle\nabla_{\nabla f} v, \nabla g\right\rangle=\langle\nabla\langle v, \nabla g\rangle, \nabla f\rangle-$ $\operatorname{Hess}(g)(v, \nabla f)$. It holds that $\operatorname{TestV}(\mathbf{X})$ is contained in $W_{C}^{1,2}(T \mathbf{X})$, but it is not known whether it is dense therein. For this reason, the space $H_{C}^{1,2}(T \mathrm{X})$ is defined as the closure of $\operatorname{TestV}(\mathbf{X})$ in $W_{C}^{1,2}(T \mathbf{X})$.

Let $v \in W_{C}^{1,2}(T \mathrm{X})$ and notice that the map from $L^{0}(T \mathrm{X})$ to $\mathrm{e}_{t}^{*} L^{0}(T \mathrm{X})$ defined by

$$
w \mapsto \mathrm{e}_{t}^{*}\left(\nabla_{w} v\right)
$$

satisfies

$$
\left|\mathrm{e}_{t}^{*}\left(\nabla_{w} v\right)\right| \leq|\nabla v|_{\mathrm{HS}} \circ \mathrm{e}_{t}|w| \circ \mathrm{e}_{t} \quad \pi \text {-a.e. }
$$

Hence by the universal property of the pullback given in Proposition 2.9 we know that there exists a unique $L^{0}(\boldsymbol{\pi})$-linear continuous operator, which we shall call $\operatorname{Cov}(v, \cdot)$ from $\mathrm{e}_{t}^{*} L^{0}(T \mathrm{X})$ to $\mathrm{e}_{t}^{*} L^{0}(T \mathrm{X})$ such that

$$
\operatorname{Cov}\left(v, \mathrm{e}_{t}^{*} w\right)=\mathrm{e}_{t}^{*}\left(\nabla_{w} v\right), \quad \forall w \in L^{0}(T \mathbf{X})
$$

and such operator satisfies the bound

$$
|\operatorname{Cov}(v, W)| \leq|\nabla v|_{\mathrm{HS}} \circ \mathrm{e}_{t}|W| \pi \text {-a.e. }
$$

We shall be interested in such covariant differentiation along the speed of our test plan: for every $t \in[0,1]$ such that $\boldsymbol{\pi}_{t}^{\prime}$ exists we define the map $\operatorname{Cov}_{t}: W_{C}^{1,2}(T \mathrm{X}) \rightarrow$ $\mathrm{e}_{t}^{*} L^{0}(T \mathrm{X})$ as

$$
\operatorname{Cov}_{t}(v):=\operatorname{Cov}\left(v, \boldsymbol{\pi}_{t}^{\prime}\right) .
$$

Notice the following simple proposition.

Proposition 3.14. For every $t \in[0,1]$ such that $\boldsymbol{\pi}_{t}^{\prime}$ exists, the map $\mathrm{Cov}_{t}$ is linear and continuous from $W_{C}^{1,2}(T \mathrm{X})$ to $\mathrm{e}_{t}^{*} L^{2}(T \mathrm{X})$.

Moreover, for every $v \in W_{C}^{1,2}(T \mathrm{X})$ the (equivalence class up to a.e. equality of the) a.e. defined map $t \mapsto \operatorname{Cov}_{t}(v) \in \mathrm{e}_{t}^{*} L^{2}(T \mathrm{X})$ is an element of $\mathscr{L}^{2}(\boldsymbol{\pi})$. 
Proof. The continuity of $\mathrm{Cov}_{t}$ as map from $W_{C}^{1,2}(T \mathrm{X})$ to $\mathrm{e}_{t}^{*} L^{2}(T \mathrm{X})$ is a direct consequence of the bounds (3.11) and our assumption (2.16):

$$
\begin{aligned}
\left\|\operatorname{Cov}_{t}(v)\right\|_{\mathrm{e}_{t}^{*} L^{2}(T \mathrm{X})}^{2} & =\int\left|\operatorname{Cov}_{t}(v)\right|^{2} \mathrm{~d} \boldsymbol{\pi} \\
& \stackrel{(3.11)}{\leq} \int|\nabla v|_{\mathrm{HS}}^{2} \circ \mathrm{e}_{t}\left|\boldsymbol{\pi}_{t}^{\prime}\right|^{2} \mathrm{~d} \boldsymbol{\pi} \leq \mathrm{C}(\boldsymbol{\pi}) \mathrm{L}(\boldsymbol{\pi})^{2}\|v\|_{W_{C}^{1,2}(T \mathrm{X})}^{2} .
\end{aligned}
$$

Thanks to this bound, to conclude it is sufficient to show that for any $v \in W_{C}^{1,2}(T \mathrm{X})$ the map $t \mapsto \operatorname{Cov}_{t}(v)=\operatorname{Cov}\left(v, \boldsymbol{\pi}_{t}^{\prime}\right)$ is a.e. equal to a Borel element of $\operatorname{VF}(\boldsymbol{\pi})$. Taking into account that $t \mapsto \boldsymbol{\pi}_{t}^{\prime} \in \mathscr{L}^{2}(\boldsymbol{\pi})$ by Proposition 3.8 , that $\operatorname{TestVF}(\boldsymbol{\pi})$ is dense in $\mathscr{L}^{2}(\boldsymbol{\pi})$, the second claim in Proposition 3.6 and the bound (3.11), we see that to conclude it is sufficient to show that $t \mapsto \operatorname{Cov}\left(v, V_{t}\right)$ is a Borel vector field in $\operatorname{VF}(\boldsymbol{\pi})$ for any $V \in \operatorname{TestVF}(\boldsymbol{\pi})$.

Thus fix such $V$, say $V_{t}=\sum_{i} \phi_{i}(t) \chi_{A_{i}} \mathrm{e}_{t}^{*} v_{i}$, and let $W_{t}=\sum_{j} \psi_{j}(t) \chi_{B_{j}} \mathrm{e}_{t}^{*} w_{j} \in$ $\operatorname{TestVF}(\boldsymbol{\pi})$ be arbitrary. Notice that since $\left|v_{i}\right|,\left|w_{j}\right| \in L^{2} \cap L^{\infty}(\mathbf{X})$, we have that $\left\langle v_{i}, \nabla_{w_{j}} v\right\rangle \in L^{1}(\mathbf{X})$ and thus by Theorem 2.11 we deduce that $t \mapsto\left\langle v_{i}, \nabla_{w_{j}} v\right\rangle \circ \mathrm{e}_{t} \in$ $L^{1}(\boldsymbol{\pi})$ is continuous for every $i, j$. Therefore

$$
t \mapsto \int\left\langle V_{t}, \operatorname{Cov}_{t}\left(v, W_{t}\right)\right\rangle \mathrm{d} \boldsymbol{\pi}=\sum_{i, j} \varphi_{i}(t) \psi_{j}(t) \int \chi_{A_{i} \cap B_{j}}\left\langle v_{i}, \nabla_{w_{j}} v\right\rangle \circ \mathrm{e}_{t} \mathrm{~d} \boldsymbol{\pi}
$$

is continuous, thus establishing, by the arbitrariness of $W$, the Borel regularity of $t \mapsto \operatorname{Cov}\left(v, V_{t}\right)$.

The 'compatibility with the metric' of the covariant derivative yields the following simple but crucial lemma.

Lemma 3.15. Let $v, w \in \operatorname{Test} \mathrm{V}(\mathrm{X})$. Then the map $t \mapsto\langle v, w\rangle \circ \mathrm{e}_{t} \in L^{2}(\boldsymbol{\pi})$, which is Lipschitz by Proposition 2.15, satisfies, for $\mathcal{L}^{1}$-a.e. $t \in[0,1]$,

$$
L^{2}(\boldsymbol{\pi})-\frac{\mathrm{d}}{\mathrm{d} t}\langle v, w\rangle \circ \mathrm{e}_{t}=\left\langle\operatorname{Cov}_{\boldsymbol{\pi}}(v)_{t}, \mathrm{e}_{t}^{*} w\right\rangle+\left\langle\mathrm{e}_{t}^{*} v, \operatorname{Cov}_{\boldsymbol{\pi}}(w)_{t}\right\rangle .
$$

Proof. Recall from [15] that it holds

$$
\mathrm{d}(\langle v, w\rangle)(z)=\left\langle\nabla_{z} v, w\right\rangle+\left\langle v, \nabla_{z} w\right\rangle \quad \mathfrak{m} \text {-a.e. } \quad \forall z \in L^{0}(T \mathbf{X}),
$$

and notice that from the defining property of pointwise norm in the pullback and by polarization we obtain that $\left\langle\mathrm{e}_{t}^{*} v_{1}, \mathrm{e}_{t}^{*} v_{2}\right\rangle=\left\langle v_{1}, v_{2}\right\rangle \circ \mathrm{e}_{t}$ for every $v_{1}, v_{2} \in L^{0}(T \mathbf{X})$. Thus we have that the identity

$$
\left(\mathrm{e}_{t}^{*} \mathrm{~d}\langle v, w\rangle\right)(Z)=\left\langle\operatorname{Cov}_{t}(v, Z), \mathrm{e}_{t}^{*} w\right\rangle+\left\langle\mathrm{e}_{t}^{*} v, \operatorname{Cov}_{t}(w, Z)\right\rangle
$$

holds for every $Z \in \mathrm{e}_{t}^{*} L^{2}(T \mathrm{X})$ of the form $Z_{t}=\mathrm{e}_{t}^{*} z$ for some $z \in L^{2}(T \mathrm{X})$. Since both sides of this identity are $L^{\infty}(\boldsymbol{\pi})$-linear and continuous in $Z$, we see that $(3.13)$ holds for generic $Z \in \mathrm{e}_{t}^{*} L^{2}(T \mathrm{X})$. The conclusion comes picking $Z=\boldsymbol{\pi}_{t}^{\prime}$ and recalling Proposition 2.15.

We now want to introduce a new differential operator, initially defined only on $\operatorname{TestVF}(\boldsymbol{\pi})$ and then extended to more general vector fields. To this aim, the following lemma will be useful. 
Lemma 3.16. Let $\left(\varphi_{i}\right),\left(\psi_{j}\right) \subset \operatorname{LIP}([0,1])$, let $\left(A_{i}\right),\left(B_{j}\right)$ Borel partitions of $\Gamma(\mathrm{X})$, and let $\left(v_{i}\right),\left(w_{j}\right) \subset \operatorname{Test} \mathrm{V}(\mathrm{X})$, where $i=1, \ldots, n$ and $j=1, \ldots, m$. Assume that

$$
\sum_{i} \chi_{A_{i}} \varphi_{i}(t) \mathrm{e}_{t}^{*} v_{i}=\sum_{j} \chi_{B_{j}} \psi_{j}(t) \mathrm{e}_{t}^{*} w_{j} \quad \text { for every } t \in[0,1]
$$

Then for a.e. $t$ it holds:

$$
\begin{aligned}
\sum_{i} \chi_{A_{i}} \varphi_{i}^{\prime}(t) \mathrm{e}_{t}^{*} v_{i} & =\sum_{j} \chi_{B_{j}} \psi_{j}^{\prime}(t) \mathrm{e}_{t}^{*} w_{j} \\
\sum_{i} \chi_{A_{i}} \varphi_{i}(t) \operatorname{Cov}_{t}\left(v_{i}\right) & =\sum_{j} \chi_{B_{j}} \psi_{j}(t) \operatorname{Cov}_{t}\left(w_{j}\right) .
\end{aligned}
$$

Proof. For the first in (3.15) we notice that our assumption (3.14) and Proposition 3.9 yield that $\sum_{i} \chi_{A_{i} \times[0,1]}(\cdot, t) \varphi_{i}(t) \mathrm{e}^{*} v_{i}=\sum_{j} \chi_{B_{j} \times[0,1]}(\cdot, t) \psi_{j}(t) \mathrm{e}^{*} w_{j}$ as elements in $\mathrm{e}^{*} L^{2}(T \mathrm{X}) \sim \mathscr{L}^{2}(\boldsymbol{\pi})$, thus we can differentiate in time and conclude using again Proposition 3.9.

For the second in (3.15) start noticing that our assumption (3.14) and the very definition of pullback imply that for any $i, j$ and every $t \in[0,1]$ it holds $\chi_{C} \varphi_{i}(t) v_{i}=\chi_{C} \psi_{j}(t) w_{j}$, where $C:=\left\{\mathrm{d}\left(\mathrm{e}_{t}\right)_{*}\left(\chi_{A_{i} \cap B_{j}} \boldsymbol{\pi}\right) / \mathrm{d}\left(\mathrm{e}_{t}\right)_{*} \boldsymbol{\pi}>0\right\}$. This identity and the locality of the covariant derivative give that $\chi_{C} \varphi_{i}(t) \nabla_{z} v_{i}=$ $\chi_{C} \psi_{j}(t) \nabla_{z} w_{j}$ for every $z \in L^{2}(T \mathrm{X})$. Applying the pullback map on both sides and noticing that $\chi_{C} \circ \mathrm{e}_{t} \geq \chi_{A_{i} \cap B_{j}}$ we deduce that

$$
\chi_{A_{i} \cap B_{j}} \varphi_{i}(t) \operatorname{Cov}\left(v_{i}, Z\right)=\chi_{A_{i} \cap B_{j}} \psi_{j}(t) \operatorname{Cov}\left(w_{j}, Z\right)
$$

for every $Z$ of the form $Z_{t}=\mathrm{e}_{t}^{*} z$. From the $L^{\infty}(\boldsymbol{\pi})$-linearity in $Z$ of both sides and the arbitrariness of $i, j$ the conclusion follows.

We can now define the convective derivative of test vector fields.

Definition 3.17 (Convective derivative along a test plan). We define the convective derivative operator $\widetilde{\mathrm{D}}_{\boldsymbol{\pi}}: \operatorname{TestVF}(\boldsymbol{\pi}) \rightarrow \mathscr{L}^{2}(\boldsymbol{\pi})$ as follows: to the element $V \in \operatorname{TestVF}(\boldsymbol{\pi})$, of the form $V_{t}=\sum_{i=1}^{n} \varphi_{i}(t) \chi_{A_{i}} \mathrm{e}_{t}^{*} v_{i}$, we associate the vector field $\widetilde{\mathrm{D}}_{\boldsymbol{\pi}} V \in \mathscr{L}^{2}(\boldsymbol{\pi})$ given by

$$
\left(\widetilde{\mathrm{D}}_{\pi} V\right)_{t}:=\sum_{i=1}^{n} \chi_{A_{i}}\left(\varphi_{i}^{\prime}(t) \mathrm{e}_{t}^{*} v_{i}+\varphi_{i}(t) \operatorname{Cov}_{t}\left(v_{i}\right)\right) \quad \text { for } \mathcal{L}^{1} \text {-a.e. } t \in[0,1] .
$$

For the sake of simplicity, we will briefly write $\widetilde{\mathrm{D}}_{\boldsymbol{\pi}} V_{t}$ instead of $\left(\widetilde{\mathrm{D}}_{\boldsymbol{\pi}} V\right)_{t}$.

Notice that Lemma 3.16 ensures that the right-hand side of (3.16) depends only on $V$ and not on the way we write it as $V_{t}=\sum_{i=1}^{n} \varphi_{i}(t) \chi_{A_{i}} \mathrm{e}_{t}^{*} v_{i}$. The fact that the right-hand side of (3.16) defines a Borel vector field in $\operatorname{VF}(\boldsymbol{\pi})$ follows directly from Proposition 3.14 ; to see that it belongs to $\mathscr{L}^{2}(\boldsymbol{\pi})$ notice that $(t \mapsto$ $\left.\mathrm{e}_{t}^{*} v_{i}\right), \operatorname{Cov}_{\boldsymbol{\pi}}\left(v_{i}\right) \in \mathscr{L}^{2}(\boldsymbol{\pi})$ for every $i$ and that the $\varphi_{i}$ 's are Lipschitz.

Therefore the definition is well posed and is then clear that $\widetilde{\mathrm{D}}_{\boldsymbol{\pi}}$ is a linear operator. 
The convective derivative has the following simple and crucial property, which is a direct consequence of Lemma 3.15.

Proposition 3.18. Let $V, W \in \operatorname{TestVF}(\boldsymbol{\pi})$. Then the map $t \mapsto\left\langle V_{t}, W_{t}\right\rangle \in L^{2}(\boldsymbol{\pi})$ is Lipschitz and satisfies

$$
L^{2}(\boldsymbol{\pi})-\frac{\mathrm{d}}{\mathrm{d} t}\left\langle V_{t}, W_{t}\right\rangle=\left\langle\widetilde{\mathrm{D}}_{\boldsymbol{\pi}} V_{t}, W_{t}\right\rangle+\left\langle V_{t}, \widetilde{\mathrm{D}}_{\boldsymbol{\pi}} W_{t}\right\rangle \quad \text { for } \mathcal{L}^{1} \text {-a.e. } t \in[0,1] .
$$

Proof. By bilinearity, to prove (3.17) is sufficient to consider the case $V_{t}=\varphi(t) \chi_{A} \mathrm{e}_{t}^{*} v$ and $W_{t}=\psi(t) \chi_{B} \mathrm{e}_{t}^{*} w$ for $v, w \in \operatorname{TestV}(\mathrm{X})$. Lemma 3.15 ensures that $t \mapsto$ $\left\langle\mathrm{e}_{t}^{*} v, \mathrm{e}_{t}^{*} w\right\rangle=\langle v, w\rangle \circ \mathrm{e}_{t} \in L^{2}(\boldsymbol{\pi})$ is Lipschitz, and it is then clear that $t \mapsto$ $\left\langle V_{t}, W_{t}\right\rangle=\chi_{A \cap B} \varphi(t) \psi(t)\left\langle\mathrm{e}_{t}^{*} v, \mathrm{e}_{t}^{*} w\right\rangle$ is also Lipschitz. The identity (3.17) now follows from (3.12) and the Leibniz rule.

This last proposition allows to 'integrate by parts' and extend the definition of convective derivative to 'Sobolev vector fields along $\boldsymbol{\pi}$ '.

Let us define the support $\operatorname{spt}(V)$ of a test vector field $V \in \operatorname{TestVF}(\boldsymbol{\pi})$ as the closure of the set of $t$ 's for which $V_{t} \neq 0$ and let us introduce the space of sections with compact support in $(0,1)$ :

$$
\operatorname{TestVF}_{c}(\boldsymbol{\pi}):=\{V \in \operatorname{TestVF}(\boldsymbol{\pi}): \operatorname{spt}(V) \subseteq(0,1)\} .
$$

A simple cut-off argument shows that $\operatorname{TestVF}_{c}(\boldsymbol{\pi})$ is $\mathscr{L}^{2}(\boldsymbol{\pi})$-dense in $\operatorname{TestVF}(\boldsymbol{\pi})$ and hence in $\mathscr{L}^{2}(\boldsymbol{\pi})$.

Definition 3.19 (The space $\mathscr{W}^{1,2}(\boldsymbol{\pi})$ ). The Sobolev space $\mathscr{W}^{1,2}(\boldsymbol{\pi})$ is the vector subspace of $\mathscr{L}^{2}(\boldsymbol{\pi})$ consisting of all those $V \in \mathscr{L}^{2}(\boldsymbol{\pi})$ such that there exists $Z \in$ $\mathscr{L}^{2}(\boldsymbol{\pi})$ satisfying

(3.18) $\int_{0}^{1} \int\left\langle V_{t}, \widetilde{\mathrm{D}}_{\boldsymbol{\pi}} W_{t}\right\rangle \mathrm{d} \boldsymbol{\pi} \mathrm{d} t=-\int_{0}^{1} \int\left\langle Z_{t}, W_{t}\right\rangle \mathrm{d} \boldsymbol{\pi} \mathrm{d} t \quad$ for every $W \in \operatorname{TestVF}_{c}(\boldsymbol{\pi})$.

In this case the section $Z$, whose uniqueness is granted by density of $\operatorname{TestVF}_{c}(\boldsymbol{\pi})$ in $\mathscr{L}^{2}(\boldsymbol{\pi})$, can be unambiguously denoted by $\mathrm{D}_{\boldsymbol{\pi}} V$ and called convective derivative of $V$. We endow $\mathscr{W}^{1,2}(\boldsymbol{\pi})$ with the norm $\|\cdot\|_{\mathscr{W}^{1,2}(\boldsymbol{\pi})}$, defined by

$$
\|V\|_{\mathscr{W}^{1,2}(\boldsymbol{\pi})}:=\sqrt{\|V\|_{\mathscr{L}^{2}(\boldsymbol{\pi})}^{2}+\left\|\mathrm{D}_{\boldsymbol{\pi}} V\right\|_{\mathscr{L}^{2}(\boldsymbol{\pi})}^{2}} \quad \text { for every } V \in \mathscr{W}^{1,2}(\boldsymbol{\pi}) .
$$

This choice of terminology is consistent with that of Definition 3.17:

Proposition 3.20. Let $V \in \operatorname{TestVF}(\boldsymbol{\pi})$. Then $V \in \mathscr{W}^{1,2}(\boldsymbol{\pi})$ and $\mathrm{D}_{\boldsymbol{\pi}} V=\widetilde{\mathrm{D}}_{\boldsymbol{\pi}} V$.

Proof. Fix $W \in \operatorname{TestVF}_{c}(\boldsymbol{\pi})$. We know from Proposition 3.18 that $[0,1] \ni t \mapsto$ $\int\left\langle V_{t}, W_{t}\right\rangle \mathrm{d} \boldsymbol{\pi}$ is an absolutely continuous function, so that (3.17) gives, after integration, that

$0=\int\left\langle V_{1}, W_{1}\right\rangle \mathrm{d} \boldsymbol{\pi}-\int\left\langle V_{0}, W_{0}\right\rangle \mathrm{d} \boldsymbol{\pi}=\int_{0}^{1} \int\left\langle\widetilde{\mathrm{D}}_{\boldsymbol{\pi}} V_{t}, W_{t}\right\rangle \mathrm{d} \boldsymbol{\pi} \mathrm{d} t+\int_{0}^{1} \int\left\langle V_{t}, \widetilde{\mathrm{D}}_{\boldsymbol{\pi}} W_{t}\right\rangle \mathrm{d} \boldsymbol{\pi} \mathrm{d} t$.

This proves that $V$ satisfies (3.18) with $Z=\widetilde{\mathrm{D}}_{\boldsymbol{\pi}} V$. 
Proposition 3.21 (Basic properties of $\mathscr{W}^{1,2}(\boldsymbol{\pi})$ ). The following hold:

(i) $\mathrm{D}_{\boldsymbol{\pi}}$ is a closed operator from $\mathscr{L}^{2}(\boldsymbol{\pi})$ into itself, i.e., its graph is closed in the product space $\mathscr{L}^{2}(\boldsymbol{\pi}) \times \mathscr{L}^{2}(\boldsymbol{\pi})$.

(ii) $\mathscr{W}^{1,2}(\boldsymbol{\pi})$ is a separable Hilbert space.

(iii) Let $V, Z \in \mathscr{L}^{2}(\boldsymbol{\pi})$. Then $V \in \mathscr{W}^{1,2}(\boldsymbol{\pi})$ and $Z=\mathrm{D}_{\boldsymbol{\pi}} V$ if and only if for every $W \in \operatorname{TestVF}(\boldsymbol{\pi})$ the map $t \mapsto\left\langle V_{t}, W_{t}\right\rangle$ belongs to $W^{1,1}\left([0,1], L^{1}(\boldsymbol{\pi})\right)$ with derivative given by

$$
\frac{\mathrm{d}}{\mathrm{d} t}\left\langle V_{t}, W_{t}\right\rangle=\left\langle V_{t}, \mathrm{D}_{\pi} W_{t}\right\rangle+\left\langle Z_{t}, W_{t}\right\rangle \text { a.e. } t .
$$

Proof. (i) Let $\left(V_{n}\right) \subseteq \mathscr{W}^{1,2}(\boldsymbol{\pi})$ be a sequence such that $V_{n} \rightarrow V$ and $\mathrm{D}_{\boldsymbol{\pi}} V_{n} \rightarrow Z$ in $\mathscr{L}^{2}(\boldsymbol{\pi})$ for some $V, Z \in \mathscr{L}^{2}(\boldsymbol{\pi})$. Then for arbitrary $W \in \operatorname{TestVF}_{c}(\boldsymbol{\pi})$ we have

$$
\begin{aligned}
\int_{0}^{1} \int\left\langle V_{t}, \mathrm{D}_{\pi} W_{t}\right\rangle \mathrm{d} \boldsymbol{\pi} \mathrm{d} t & =\lim _{n \rightarrow \infty} \int_{0}^{1} \int\left\langle V_{t}^{n}, \mathrm{D}_{\boldsymbol{\pi}} W_{t}\right\rangle \mathrm{d} \boldsymbol{\pi} \mathrm{d} t \\
& =-\lim _{n \rightarrow \infty} \int_{0}^{1} \int\left\langle\mathrm{D}_{\boldsymbol{\pi}} V_{t}^{n}, W_{t}\right\rangle \mathrm{d} \boldsymbol{\pi} \mathrm{d} t=-\int_{0}^{1} \int\left\langle Z_{t}, W_{t}\right\rangle \mathrm{d} \boldsymbol{\pi} \mathrm{d} t
\end{aligned}
$$

proving that $V \in \mathscr{W}^{1,2}(\boldsymbol{\pi})$ with $\mathrm{D}_{\boldsymbol{\pi}} V=Z$, which was the claim.

(ii) Consequence of what just proved and the fact that the map

$$
\mathscr{W}^{1,2}(\boldsymbol{\pi}) \ni V \quad \mapsto \quad\left(V, \mathrm{D}_{\boldsymbol{\pi}} V\right) \in \mathscr{L}^{2}(\boldsymbol{\pi}) \times \mathscr{L}^{2}(\boldsymbol{\pi})
$$

is an isometry, provided we endow $\mathscr{L}^{2}(\boldsymbol{\pi}) \times \mathscr{L}^{2}(\boldsymbol{\pi})$, with the (separable, by Proposition 3.7) norm $\|(V, Z)\|^{2}:=\|V\|_{\mathscr{L}^{2}(\boldsymbol{\pi})}^{2}+\|Z\|_{\mathscr{L}^{2}(\boldsymbol{\pi})}^{2}$.

(iii) The 'if' trivially follows from (3.19) by integration. For the 'only if', fix $W \in \operatorname{TestVF}(\boldsymbol{\pi})$ and let $\varphi \in C_{c}^{1}(0,1)$ and $\Gamma \subset \Gamma(\mathrm{X})$ Borel. Then $t \mapsto \varphi(t) \chi_{\Gamma} W_{t}$ is in $\operatorname{TestVF}_{c}(\boldsymbol{\pi})$ and a direct computation shows that $\mathrm{D}_{\boldsymbol{\pi}}\left(\varphi \chi_{\Gamma} W\right)_{t}=\varphi^{\prime}(t) \chi_{\Gamma} W_{t}+$ $\varphi(t) \chi_{\Gamma} \mathrm{D}_{\pi} W_{t}$. Hence writing the defining property (3.18) with $\varphi \chi_{\Gamma} W$ in place of $W$ we obtain, after rearrangement, that

$$
\int_{0}^{1} \varphi^{\prime}(t) \int_{\Gamma}\left\langle V_{t}, W_{t}\right\rangle \mathrm{d} \boldsymbol{\pi} \mathrm{d} t=-\int_{0}^{1} \varphi(t) \int_{\Gamma}\left\langle V_{t}, \mathrm{D}_{\boldsymbol{\pi}} W_{t}\right\rangle+\left\langle Z_{t}, W_{t}\right\rangle \mathrm{d} \boldsymbol{\pi} \mathrm{d} t .
$$

The arbitrariness of $\varphi, \Gamma$ and Proposition 2.3 yield the claim.

We just proved that $\operatorname{TestVF}(\boldsymbol{\pi})$ is contained in $\mathscr{W}^{1,2}(\boldsymbol{\pi})$, but we don't know if it is dense. Hence the following definition is meaningful.

Definition 3.22 (The space $\left.\mathscr{H}^{1,2}(\boldsymbol{\pi})\right) \cdot \mathscr{H}^{1,2}(\boldsymbol{\pi})$ is the $\mathscr{W}^{1,2}(\boldsymbol{\pi})$-closure of $\operatorname{TestVF}(\boldsymbol{\pi})$.

Clearly, $\mathscr{H}^{1,2}(\boldsymbol{\pi})$ is a separable Hilbert space. A key feature of elements of $\mathscr{H}^{1,2}(\boldsymbol{\pi})$ is that they admit a continuous representative (much like Sobolev functions on the interval): 
Theorem 3.23. The inclusion $\operatorname{TestVF}(\boldsymbol{\pi}) \hookrightarrow \mathscr{C}(\boldsymbol{\pi})$ uniquely extends to a linear continuous and injective operator $\iota: \mathscr{H}^{1,2}(\boldsymbol{\pi}) \rightarrow \mathscr{C}(\boldsymbol{\pi})$.

Proof. We claim that

$$
\|V\|_{\mathscr{C}(\boldsymbol{\pi})} \leq \sqrt{2}\|V\|_{\mathscr{W}^{1,2}(\boldsymbol{\pi})}, \quad \forall V \in \operatorname{TestVF}(\boldsymbol{\pi}) .
$$

By the density of $\operatorname{TestVF}(\boldsymbol{\pi})$ in $\mathscr{H}^{1,2}(\boldsymbol{\pi})$ this will be enough to obtain the existence of $\iota$. Thus let $V \in \operatorname{TestVF}(\boldsymbol{\pi})$, pick $W=V$ in $(3.17)$ and integrate in $\left[t_{1}, t_{2}\right] \subset[0,1]$ and w.r.t. $\boldsymbol{\pi}$ to obtain

$$
\begin{aligned}
\left|\llbracket V \rrbracket_{t_{2}}^{2}-\llbracket V \rrbracket_{t_{1}}^{2}\right| & =2\left|\int_{t_{1}}^{t_{2}} \int\left\langle V_{t}, \mathrm{D}_{\boldsymbol{\pi}} V_{t}\right\rangle \mathrm{d} \boldsymbol{\pi} \mathrm{d} t\right| \\
& \leq 2 \int_{t_{1}}^{t_{2}} \int\left|V_{t}\right|\left|\mathrm{D}_{\boldsymbol{\pi}} V_{t}\right| \mathrm{d} \boldsymbol{\pi} \mathrm{d} t \leq\|V\|_{\mathscr{L}^{2}(\boldsymbol{\pi})}^{2}+\left\|\mathrm{D}_{\boldsymbol{\pi}} V\right\|_{\mathscr{L}^{2}(\boldsymbol{\pi})}^{2} .
\end{aligned}
$$

Hence for any $t \in[0,1]$ one has

$$
\llbracket V \rrbracket_{t}^{2}=\int_{0}^{1} \llbracket V \rrbracket_{t}^{2} \mathrm{~d} s \leq \int_{0}^{1}\left|\llbracket V \rrbracket_{t}^{2}-\llbracket V \rrbracket_{s}^{2}\right| \mathrm{d} s+\|V\|_{\mathscr{L}^{2}(\boldsymbol{\pi})}^{2} \leq 2\|V\|_{\mathscr{W} 1,2}^{2}(\boldsymbol{\pi}),
$$

which is our claim (3.20).

To prove injectivity, let $V \in \mathscr{H}^{1,2}(\boldsymbol{\pi})$ be such that $\iota(V)=0$. Choose a sequence $\left(V_{n}\right) \subseteq \operatorname{TestVF}(\boldsymbol{\pi})$ which is $\mathscr{W}^{1,2}(\boldsymbol{\pi})$-converging to $V$ and notice that, up to pass to a subsequence and using Proposition 3.6, we can assume that $V_{t}^{n} \rightarrow V_{t}$ for $\mathcal{L}^{1}$-a.e. $t \in[0,1]$. By continuity of the operator $\iota$, one also has $\left\|V^{n}\right\|_{\mathscr{C}(\boldsymbol{\pi})}=$ $\left\|\iota\left(V^{n}\right)-\iota(V)\right\|_{\mathscr{C}(\boldsymbol{\pi})} \rightarrow 0$ and thus in particular $V_{t}^{n} \rightarrow 0$ for all $t \in[0,1]$. Therefore $V_{t}=0$ for $\mathcal{L}^{1}$-a.e. $t \in[0,1]$, yielding the required injectivity of $\iota$.

Whenever we will consider an element $V$ of $\mathscr{H}^{1,2}(\boldsymbol{\pi})$, we will always implicitly refer to its unique continuous representative $\iota(V) \in \mathscr{C}(\boldsymbol{\pi})$.

Among the several properties of the test sections that can be carried over to the elements of $\mathscr{H}^{1,2}(\boldsymbol{\pi})$, the most important one is the Leibniz formula for the convective derivatives:

Proposition 3.24 (Leibniz formula for $\mathrm{D}_{\boldsymbol{\pi}}$ ). Let $V \in \mathscr{W}^{1,2}(\boldsymbol{\pi})$ and $W \in \mathscr{H}^{1,2}(\boldsymbol{\pi})$. Then the function $t \mapsto\left\langle V_{t}, W_{t}\right\rangle$ is in $W^{1,1}\left([0,1], L^{1}(\boldsymbol{\pi})\right)$ and its derivative is given by

$$
\frac{\mathrm{d}}{\mathrm{d} t}\left\langle V_{t}, W_{t}\right\rangle=\left\langle\mathrm{D}_{\boldsymbol{\pi}} V_{t}, W_{t}\right\rangle+\left\langle V_{t}, \mathrm{D}_{\boldsymbol{\pi}} W_{t}\right\rangle \quad \text { for } \mathcal{L}^{1} \text {-a.e. } t \in[0,1] .
$$

Proof. For $W \in \operatorname{TestVF}(\boldsymbol{\pi})$ the claim is a direct consequence of point (iii) in Proposition 3.21. The general case can be achieved by approximation noticing that the simple inequalities

$$
\begin{aligned}
&\left\|\left\langle V_{t}, W_{t}\right\rangle\right\|_{L^{1}\left(\boldsymbol{\pi} \times \mathcal{L}_{1}\right)} \leq\|V\|_{\mathscr{L}^{2}(\boldsymbol{\pi})}\|W\|_{\mathscr{L}^{2}(\boldsymbol{\pi})}, \\
&\left\|\left\langle\mathrm{D}_{\boldsymbol{\pi}} V_{t}, W_{t}\right\rangle+\left\langle V_{t}, \mathrm{D}_{\boldsymbol{\pi}} W_{t}\right\rangle\right\|_{L^{1}\left(\boldsymbol{\pi} \times \mathcal{L}_{1}\right)} \leq 2\|V\|_{\mathscr{W}^{1,2}(\boldsymbol{\pi})}\|W\|_{\mathscr{W}^{1,2}(\boldsymbol{\pi})},
\end{aligned}
$$

allow to pass to the limit in the distributional formulation of $\frac{\mathrm{d}}{\mathrm{d} t}\left\langle V_{t}, W_{t}\right\rangle$ as $W$ varies in $\mathscr{H}^{1,2}(\boldsymbol{\pi})$. 
In the next proposition we collect some examples of elements of $\mathscr{H}^{1,2}(\boldsymbol{\pi})$.

Proposition 3.25. Let $\boldsymbol{\pi}$ be a Lipschitz test plan. Then:

(i) For every $w \in H_{C}^{1,2}(T \mathrm{X})$ the vector field $t \mapsto W_{t}:=\mathrm{e}_{t}^{*} w$ belongs to $\mathscr{H}^{1,2}(\boldsymbol{\pi})$ and

$$
\mathrm{D}_{\pi} W_{t}=\operatorname{Cov}_{t}(w) \quad \text { a.e. } t .
$$

(ii) Let $W \in \mathscr{H}^{1,2}(\boldsymbol{\pi})$ be such that $|W|,\left|\mathrm{D}_{\boldsymbol{\pi}} W\right| \in L^{\infty}\left(\boldsymbol{\pi} \times \mathcal{L}_{1}\right)$ and let $a \in$ $W^{1,2}\left([0,1], L^{2}(\boldsymbol{\pi})\right)$. Then $a W \in \mathscr{H}^{1,2}(\boldsymbol{\pi})$ with

$$
\mathrm{D}_{\pi}(a W)_{t}=a_{t}^{\prime} W_{t}+a_{t} \mathrm{D}_{\boldsymbol{\pi}} W_{t} \quad \text { a.e. } t .
$$

Moreover, if $W \in \mathscr{C}(\boldsymbol{\pi})$ and $a \in A C^{2}\left([0,1], L^{2}(\boldsymbol{\pi})\right)$, then $a W \in \mathscr{C}(\boldsymbol{\pi})$.

Proof. (i) If $w \in \operatorname{TestV}(\mathbf{X})$ we have that $W \in \operatorname{TestVF}(\boldsymbol{\pi})$ by definition and in this case formula (3.21) holds by the definition (3.16) and Proposition 3.20. The general case can then be obtained by approximating $w$ with vector fields in TestV(X) w.r.t. the $W_{C}^{1,2}(T \mathrm{X})$ topology, using the bounds

$$
\begin{gathered}
\int_{0}^{1} \int\left|\mathrm{e}_{t}^{*}(v)\right|^{2} \mathrm{~d} \boldsymbol{\pi} \mathrm{d} t=\int_{0}^{1} \int|v|^{2} \circ \mathrm{e}_{t} \mathrm{~d} \boldsymbol{\pi} \mathrm{d} t \leq \mathrm{C}(\boldsymbol{\pi})\|v\|_{W_{C}^{1,2}(T \mathrm{X})}^{2}, \\
\int_{0}^{1} \int\left|\operatorname{Cov}_{\boldsymbol{\pi}}(v)_{t}\right|^{2} \mathrm{~d} \boldsymbol{\pi} \mathrm{d} t \stackrel{(3.11)}{\leq} \mathrm{C}(\boldsymbol{\pi}) \mathrm{L}(\boldsymbol{\pi})^{2}\|v\|_{W_{C}^{1,2}(T \mathrm{X})}^{2}
\end{gathered}
$$

and recalling the closure of $\mathrm{D}_{\boldsymbol{\pi}}$.

(ii) The claim about continuity is obvious, so we concentrate on the other one. Assume at first that $a$ belongs to the space $\mathcal{A}$ defined as

$$
\mathcal{A}:=\left\{\sum_{i=1}^{n} \varphi_{i} \chi_{E_{i}}: n \in \mathbb{N}, \varphi_{i} \in \operatorname{LIP}([0,1]),\left(E_{i}\right) \text { Borel partition of } \Gamma(\mathrm{X})\right\}
$$

and that $W \in \operatorname{TestVF}(\boldsymbol{\pi})$. In this case $a W$ belongs to $\operatorname{TestVF}(\boldsymbol{\pi})$ as well and formula (3.22) is a direct consequence of the definitions. Then using the trivial bounds

$$
\begin{aligned}
\|a W\|_{\mathscr{L}^{2}(\boldsymbol{\pi})} & \leq\|a\|_{L^{\infty}\left(\boldsymbol{\pi} \times \mathcal{L}_{1}\right)}\|W\|_{\mathscr{L}^{2}(\boldsymbol{\pi})}, \\
\left\|a^{\prime} W+a \mathrm{D}_{\boldsymbol{\pi}} W\right\|_{\mathscr{L}^{2}(\boldsymbol{\pi})} & \leq\left(\|a\|_{L^{\infty}\left(\boldsymbol{\pi} \times \mathcal{L}_{1}\right)}+\left\|a^{\prime}\right\|_{L^{\infty}\left(\boldsymbol{\pi} \times \mathcal{L}_{1}\right)}\right)\|W\|_{\mathscr{W}^{1,2}(\boldsymbol{\pi})},
\end{aligned}
$$

the $\mathscr{W}^{1,2}(\boldsymbol{\pi})$-density of $\operatorname{TestVF}(\boldsymbol{\pi})$ in $\mathscr{H}^{1,2}(\boldsymbol{\pi})$ and the closure of $\mathrm{D}_{\boldsymbol{\pi}}$, we conclude that $a W \in \mathscr{H}^{1,2}(\boldsymbol{\pi})$ for every $a \in \mathcal{A}$ and $W \in \mathscr{H}^{1,2}(\boldsymbol{\pi})$ and that (3.22) holds in this case.

Now let $W$ be as in the assumptions and notice that we also have the bounds

$$
\begin{aligned}
\|a W\|_{\mathscr{L}^{2}(\boldsymbol{\pi})} & \leq\|a\|_{L^{2}\left([0,1], L^{2}(\boldsymbol{\pi})\right)}\||W|\|_{L^{\infty}\left(\boldsymbol{\pi} \times \mathcal{L}_{1}\right)}, \\
\left\|a^{\prime} W+a \mathrm{D}_{\boldsymbol{\pi}} W\right\|_{\mathscr{L}^{2}(\boldsymbol{\pi})} & \leq\|a\|_{W^{1,2}\left([0,1], L^{2}(\boldsymbol{\pi})\right)}\left(\||W|\|_{L^{\infty}\left(\boldsymbol{\pi} \times \mathcal{L}_{1}\right)}+\left\|\mathrm{D}_{\boldsymbol{\pi}} W \mid\right\|_{L^{\infty}\left(\boldsymbol{\pi} \times \mathcal{L}_{1}\right)}\right)
\end{aligned}
$$


therefore using again the closure of $\mathrm{D}_{\boldsymbol{\pi}}$, to conclude it is sufficient to prove that $\mathcal{A}$ is dense in $W^{1,2}\left([0,1], L^{2}(\boldsymbol{\pi})\right)$. To this aim we argue as follows: for every $n \in \mathbb{N}$, let $\left(E_{i}^{n}\right)_{i \in \mathbb{N}}$ be a Borel partition of $\operatorname{supp}(\boldsymbol{\pi}) \subset \Gamma(\mathrm{X})$ made of sets with positive $\boldsymbol{\pi}$ measure and diameter $\leq 1 / n$. Then for every $n, N \in \mathbb{N}$, let $P_{n}^{N}: L^{2}(\boldsymbol{\pi}) \rightarrow L^{2}(\boldsymbol{\pi})$ be defined by

$$
P_{n}^{N}(f):=\sum_{i=1}^{N} \chi_{E_{i}^{n}} \frac{1}{\pi\left(E_{i}^{n}\right)} \int_{E_{i}^{n}} f \mathrm{~d} \boldsymbol{\pi} .
$$

It is clear that $P_{n}^{N}$ has operator norm $\leq 1$ for every $n, N \in \mathbb{N}$, and an application of the dominated convergence theorem shows that

$$
\lim _{n} \lim _{N} P_{n}^{N}(f)=f
$$

for every $f \in C_{b}(\Gamma(\mathrm{X}))$, the limits being intended in $L^{2}(\boldsymbol{\pi})$. Therefore (3.23) also holds for every $f \in L^{2}(\boldsymbol{\pi})$. The linearity and continuity of $P_{n}^{N}$ also grants that if $t \mapsto a_{t}$ belongs to $W^{1,2}\left([0,1], L^{2}(\boldsymbol{\pi})\right)$, then also $t \mapsto P_{n}^{N}(a)_{t}:=P_{n}^{N}\left(a_{t}\right)$ belongs to $W^{1,2}\left([0,1], L^{2}(\boldsymbol{\pi})\right)$, with

$$
\left(P_{n}^{N}(a)\right)_{t}^{\prime}=P_{n}^{N}\left(a_{t}^{\prime}\right) \text { a.e. } t .
$$

All these considerations imply that

$$
\lim _{n} \lim _{N} P_{n}^{N}(a)=a \quad \text { in } W^{1,2}\left([0,1], L^{2}(\boldsymbol{\pi})\right)
$$

for every $a \in W^{1,2}\left([0,1], L^{2}(\boldsymbol{\pi})\right)$, and thus to conclude it is sufficient to prove that $P_{n}^{N}(a)$ belongs to the $W^{1,2}\left([0,1], L^{2}(\boldsymbol{\pi})\right)$-closure of $\mathcal{A}$ for every $n, N \in \mathbb{N}$ and $a \in W^{1,2}\left([0,1], L^{2}(\boldsymbol{\pi})\right)$.

It is clear by construction and (3.24) that $P_{n}^{N}(a)=\sum_{i=1}^{N} g_{i} \chi_{E_{i}^{n}}$ for some $g_{i} \in W^{1,2}([0,1])$. Now for every $i=1, \ldots, N$ find $\left(g_{i, j}\right) \subset \operatorname{LIP}([0,1])$ which $W^{1,2}([0,1])$-converges to $g_{i}$, and notice that

$$
\left\|\sum_{i=1}^{N}\left(g_{i, j}-g_{i}\right) \chi_{E_{i}^{n}}\right\|_{W^{1,2}\left([0,1], L^{2}(\pi)\right)}^{2}=\sum_{i=1}^{N} \boldsymbol{\pi}\left(E_{i}^{n}\right)\left\|g_{i, j}-g_{i}\right\|_{W^{1,2}([0,1])}^{2} \rightarrow 0
$$

as $j \rightarrow \infty$. Since $\sum_{i=1}^{N} g_{i, j} \chi_{E_{i}^{n}} \in \mathcal{A}$ for every $j$, the proof is finished.

\section{Parallel transport on RCD spaces}

\subsection{Definition and basic properties of parallel transport}

4.1.1. Definition and uniqueness. We shall frequently use the fact that, since $\mathscr{H}^{1,2}(\boldsymbol{\pi})$ is continuously embedded into $\mathscr{C}(\boldsymbol{\pi})$ by Theorem 3.23 , any vector field $V \in \mathscr{H}^{1,2}(\boldsymbol{\pi})$ has pointwise values $V_{t} \in \mathrm{e}_{t}^{*} L^{2}(T \mathrm{X})$ defined at every time $t \in[0,1]$.

Definition 4.1 (Parallel transport). Let $K \in \mathbb{R},(\mathrm{X}, \mathrm{d}, \mathfrak{m})$ an $\mathrm{RCD}(K, \infty)$ space and $\boldsymbol{\pi}$ be a Lipschitz test plan on X. A parallel transport along $\boldsymbol{\pi}$ is an element $V \in \mathscr{H}^{1,2}(\boldsymbol{\pi})$ such that $\mathrm{D}_{\boldsymbol{\pi}} V=0$. 
The linearity of the requirement $\mathrm{D}_{\pi} V=0$ ensures that the set of parallel transports along $\boldsymbol{\pi}$ is a vector space. From Proposition 3.24 we deduce the following simple but crucial result.

Proposition 4.2 (Norm preservation). Let $V$ be a parallel transport along the Lipschitz test plan $\boldsymbol{\pi}$. Then $t \mapsto\left|V_{t}\right|^{2} \in L^{1}(\boldsymbol{\pi})$ is constant.

Proof. We know from Corollary 3.13 that $t \mapsto\left|V_{t}\right|^{2} \in L^{1}(\boldsymbol{\pi})$ is continuous. Hence the choice $W=V$ in Proposition 3.24 tells that such map is absolutely continuous with derivative given by

$$
\frac{\mathrm{d}}{\mathrm{d} t}\left|V_{t}\right|^{2}=2\left\langle\mathrm{D}_{\pi} V_{t}, V_{t}\right\rangle=0, \quad \text { a.e. } t .
$$

This is sufficient to conclude.

Linearity and norm preservation imply uniqueness:

Corollary 4.3 (Uniqueness of parallel transport). Let $\boldsymbol{\pi}$ be a Lipschitz test plan, and let $V_{1}$ and $V_{2}$ be two parallel transports along it such that for some $t_{0} \in[0,1]$ it holds $V_{1, t_{0}}=V_{2, t_{0}}$. Then $V_{1}=V_{2}$.

Proof. Since $\mathrm{D}_{\pi}\left(V_{1}-V_{2}\right)=\mathrm{D}_{\pi} V_{1}-\mathrm{D}_{\pi} V_{2}=0$, we have that $V_{1}-V_{2}$ is a parallel transport and by assumption we know that $\left|V_{1, t_{0}}-V_{2, t_{0}}\right|=0 \pi$-a.e.. Thus Proposition 4.2 above grants that for every $t \in[0,1]$ it holds $\left|V_{1, t}-V_{2, t}\right|=0 \pi$-a.e., i.e., that $V_{1, t}=V_{2, t}$.

Remark 4.4. We emphasize that the norm preservation property is a consequence of the Leibniz formula in Proposition 3.24. We do not know if such formula holds for $V, W \in \mathscr{W}^{1,2}(\boldsymbol{\pi})$ and this is why we defined the parallel transport as an element of $\mathscr{H}^{1,2}(\boldsymbol{\pi})$ with null convective derivative, as opposed to an element of $\mathscr{W}^{1,2}(\boldsymbol{\pi})$ with the same property.

4.1.2. Some consequences of existence of parallel transport. In this section we assume existence of parallel transport along some/all Lipschitz test plans and see what can be derived from such assumption.

It will be convenient to recall the concept of basis of a module, referring to [15] for a more detailed discussion. Let $\mu$ be a Borel measure on a Polish space $Y$, let $\mathscr{M}$ be a $L^{2}(\mu)$-normed module, let $v_{1}, \ldots, v_{n} \in \mathscr{M}$, and let $E \subset \mathrm{Y}$ be a Borel set. Then the $v_{i}$ 's are said to be independent on $E$ provided for any $f_{i} \in L^{\infty}(\mu)$ we have

$$
\sum_{i} f_{i} v_{i}=0 \Rightarrow f_{i}=0 \quad \mu \text {-a.e. on } E
$$

and generators of $\mathscr{M}$ on $E$ provided $L^{\infty}(\mu)$-linear combinations of the $v_{i}$ 's are dense in $\left\{\chi_{E} v: v \in \mathscr{M}\right\}$. If $v_{1}, \ldots, v_{n}$ are both independent and generators of $\mathscr{M}$ on $E$, we say that they are a basis of $\mathscr{M}$ on $E$ and in this case we say that the dimension of $\mathscr{M}$ on $E$ is $n$. 
Recall that there always exists a (unique up to $\mu$-negligible sets) Borel partition $\left(E_{i}\right)_{i \in \mathbb{N} \cup\{\infty\}}$ of $\mathrm{Y}$, called dimensional decomposition of $\mathscr{M}$, such that the dimension of $\mathscr{M}$ on $E_{i}$ is $i$ for every $i \in \mathbb{N}$ and for no Borel subset $F$ of $E_{\infty}$ with positive measure the dimension of $\mathscr{M}$ on $F$ is finite.

For a separable Hilbert module $\mathscr{H}$ on a space with finite measure $\mu$, one can always find an orthonormal basis $\left(v_{n}\right)_{n \in \mathbb{N}}$, i.e., a sequence whose $L^{\infty}$-linear combinations are dense in $\mathscr{H}$ and such that for some Borel partition $\left(E_{n}\right)_{n \in \mathbb{N} \cup\{\infty\}}$ the following hold:

$\forall n \in \mathbb{N} \cup\{\infty\}$ we have $\left\langle v_{i}, v_{j}\right\rangle=\delta_{i j} \quad \mu$-a.e. on $E_{n}, \forall i, j \in \mathbb{N}, i, j<n$,

$\forall n \in \mathbb{N}$ we have

$\left|v_{i}\right|=0 \quad \mu$-a.e. on $E_{n}, \forall i \in \mathbb{N}, i \geq n$,

and it is easily verified that if these hold, then necessarily the $E_{n}$ 's form the dimensional decomposition of $\mathscr{H}$ (the role of the assumption about finiteness of $\mu$ is to ensure that the $v_{i}$ 's are elements of $\mathscr{H}$ : their pointwise norm is in $L^{\infty}(\mu)$ and thus in general it may be not in $\left.L^{2}(\mu)\right)$.

Given such a basis and $v \in \mathscr{H}$, there are functions $a_{n} \in L^{2}(\mu)$ such that

$$
v=\sum_{n \in \mathbb{N}} a_{n} v_{n},
$$

meaning that the sequence converges absolutely in $\mathscr{H}$. A choice for the $a_{n}$ is $a_{n}:=\left\langle v, v_{n}\right\rangle$ and for any two sequences $\left(a_{n}\right),\left(\tilde{a}_{n}\right)$ for which (4.1) holds we have

$$
a_{n}=\tilde{a}_{n} \quad \mu \text {-a.e. on } E_{m}, \quad \forall m \in \mathbb{N} \cup\{\infty\}, m>n \text {. }
$$

With this said, it is easy to see that parallel transport sends bases into bases:

Proposition 4.5. Let $\boldsymbol{\pi}$ be a Lipschitz test plan such that for every $t \in[0,1]$ and $\bar{V}_{t} \in \mathrm{e}_{t}^{*} L^{2}(T \mathrm{X})$ there exists a (necessarily unique) parallel transport $V$ along $\boldsymbol{\pi}$ such that $V_{t}=\bar{V}_{t}$.

Also, let $\left(E_{n}\right)_{n \in \mathbb{N} \cup\{\infty\}}$ be the dimensional decomposition of $\mathrm{e}_{0}^{*} L^{2}(T \mathrm{X})$, let $\left(\bar{V}_{n}\right)$ $\subset \mathrm{e}_{0}^{*} L^{2}(T \mathrm{X}), n \in \mathbb{N}$, be an orthonormal basis of $\mathrm{e}_{0}^{*} L^{2}(T \mathrm{X})$, and denote by $t \mapsto V_{n, t}$ the parallel transport of $\bar{V}_{n}$ along $\boldsymbol{\pi}$.

Then, for every $t \in[0,1]$, the partition $\left(E_{n}\right)_{n \in \mathbb{N} \cup\{\infty\}}$ is also the dimensional decomposition of $\mathrm{e}_{t}^{*} L^{2}(T \mathrm{X})$ and the set $\left(V_{n, t}\right)$ is an orthonormal basis of $\mathrm{e}_{t}^{*} L^{2}(T \mathrm{X})$.

Proof. For every $t, s \in[0,1]$, consider the map sending $\bar{V} \in \mathrm{e}_{t}^{*} L^{2}(T \mathrm{X})$ to $V_{s} \in$ $\mathrm{e}_{s}^{*} L^{2}(T \mathrm{X})$, where $V \in \mathscr{H}^{1,2}(\boldsymbol{\pi})$ is the parallel transport such that $V_{t}=\bar{V}$. Proposition 4.2 ensures that this map preserves the pointwise norm. Since it is clearly linear, it is easily verified that it must be an isomorphism of $\mathrm{e}_{t}^{*} L^{2}(T \mathrm{X})$ and $\mathrm{e}_{s}^{*} L^{2}(T \mathrm{X})$.

The conclusions follow.

We shall apply this result to show that, under the same assumptions, we have $\mathscr{W}^{1,2}(\boldsymbol{\pi})=\mathscr{H}^{1,2}(\boldsymbol{\pi})$.

Proposition $4.6(\mathscr{H}=\mathscr{W})$. Let $\boldsymbol{\pi}$ be a Lipschitz test plan such that for every $t \in[0,1]$ and $\bar{V}_{t} \in \mathrm{e}_{t}^{*} L^{2}(T \mathrm{X})$ there exists a (necessarily unique) parallel transport $V$ along $\pi$ such that $V_{t}=\bar{V}_{t}$.

Then $\mathscr{H}^{1,2}(\boldsymbol{\pi})=\mathscr{W}^{1,2}(\boldsymbol{\pi})$. 
Proof. Let $V \in \mathscr{W}^{1,2}(\boldsymbol{\pi})$, let $\left(\bar{V}_{i}\right) \subset \mathrm{e}_{0}^{*} L^{2}(T \mathrm{X}), i \in \mathbb{N}$, be an orthonormal basis of $\mathrm{e}_{0}^{*} L^{2}(T \mathrm{X})$, and let $t \mapsto V_{i, t}$ the parallel transport of $\bar{V}_{i}$ along $\boldsymbol{\pi}$. Then by Proposition 4.5 above we see that

$$
V_{t}=\sum_{i \in \mathbb{N}} a_{i, t} V_{i, t}, \quad \text { where } \quad a_{i, t}:=\left\langle V_{t}, V_{i, t}\right\rangle \quad \text { a.e. } t
$$

being intended that the series converges absolutely in $\mathrm{e}_{t}^{*} L^{2}(T \mathrm{X})$ for a.e. $t$. By Proposition 3.24 , we see that $t \mapsto a_{i, t}$ is in $W^{1,1}\left([0,1], L^{1}(\boldsymbol{\pi})\right)$ with derivative given by

$$
a_{i, t}^{\prime}=\left\langle\mathrm{D}_{\pi} V_{t}, V_{i, t}\right\rangle
$$

In particular, since $\left|V_{i, t}\right| \leq 1$ we see that $a_{i, t}, a_{i, t}^{\prime} \in L^{2}\left([0,1], L^{2}(\boldsymbol{\pi})\right)$, and in turn this implies - by Proposition 2.3 - that $t \mapsto a_{i, t}$ is in $W^{1,2}\left([0,1], L^{2}(\boldsymbol{\pi})\right)$. This fact and point (ii) in Proposition 3.25 give that $\left(t \mapsto a_{i, t} V_{i, t}\right) \in \mathscr{H}^{1,2}(\boldsymbol{\pi})$ for every $i \in \mathbb{N}$, and therefore $\left(t \mapsto \sum_{i=0}^{n} a_{i, t} V_{i, t}\right) \in \mathscr{H}^{1,2}(\boldsymbol{\pi})$ for every $n \in \mathbb{N}$.

Hence to conclude it is sufficient to show that these partial sums are a $\mathscr{W}^{1,2}(\boldsymbol{\pi})$ Cauchy sequence, as then it is clear from (4.2) that the limit coincides with $V$. From (4.2) and (4.3) we have that

$$
\sum_{i \in \mathbb{N}} \iint_{0}^{1}\left|a_{i, t}\right|^{2}+\left|a_{i, t}^{\prime}\right|^{2} \mathrm{~d} t \mathrm{~d} \boldsymbol{\pi}=\|V\|_{\mathscr{L}^{2}(\boldsymbol{\pi})}^{2}+\left\|\mathrm{D}_{\boldsymbol{\pi}} V\right\|_{\mathscr{L}^{2}(\boldsymbol{\pi})}^{2}<\infty
$$

hence the conclusion follows from the identity

$$
\begin{aligned}
\left\|\sum_{i=n}^{m} a_{i} V_{i}\right\|_{\mathscr{W}^{1,2}(\boldsymbol{\pi})}^{2} & =\left\|\sum_{i=n}^{m} a_{i} V_{i}\right\|_{\mathscr{L}^{2}(\boldsymbol{\pi})}^{2}+\left\|\sum_{i=n}^{m} a_{i}^{\prime} V_{i}\right\|_{\mathscr{L}^{2}(\boldsymbol{\pi})}^{2} \\
& =\sum_{i=n}^{m} \iint_{0}^{1}\left|a_{i, t}\right|^{2}+\left|a_{i, t}^{\prime}\right|^{2} \mathrm{~d} t \mathrm{~d} \boldsymbol{\pi} .
\end{aligned}
$$

We shall now prove that if the parallel transport exists along all Lipschitz test plans, then the dimension of $\mathbf{X}$, intended here as the dimension of the tangent module, must be constant. We shall use the following simple lemma (for simplicity we state it for Hilbert modules, but in fact the same holds for general ones).

Lemma 4.7. Let $\left(\mathrm{X}, \mathrm{d}_{\mathrm{X}}, \mathfrak{m}_{\mathrm{X}}\right)$ and $\left(\mathrm{Y}, \mathrm{d}_{\mathrm{Y}}, \mathfrak{m}_{\mathrm{Y}}\right)$ be two metric measure spaces, let $\varphi: \mathrm{Y} \rightarrow \mathrm{X}$ be of bounded compression, and let $\mathscr{H}$ be a Hilbert module on $\mathrm{X}$. Then for every $E \subset \mathrm{X}$ Borel we have that the dimension of $\mathscr{H}$ on $E$ is $n$ if and only if the dimension of $\varphi^{*} \mathscr{H}$ on $\varphi^{-1}(E)$ is $n$.

Proof. From the well-posedness of the definition of dimension, we see that it is sufficient to prove the only if. Thus let $v_{0}, \ldots, v_{n-1}$ be an orthonormal basis of $\mathscr{H}$ on $E$, so in particular $\left\langle v_{i}, v_{j}\right\rangle=\delta_{i j} \mathfrak{m}_{\mathbf{X}}$-a.e. on $E$.

From the fact that $v_{0}, \ldots, v_{n-1}$ generate $\mathscr{H}$ on $E$ and the very definition of pullback, we deduce that $\varphi^{*} v_{0}, \ldots, \varphi^{*} v_{n-1}$ generate $\varphi^{*} \mathscr{H}$ on $\varphi^{-1}(E)$. 
To see that they are independent, let $f_{0}, \ldots, f_{n-1} \in L^{\infty}(\mathrm{Y})$ be such that $\sum_{i} f_{i} \varphi^{*} v_{i}=0$ on $\varphi^{-1}(E)$. Then it holds

$$
\begin{aligned}
0=\left|\sum_{i} f_{i} \varphi^{*} v_{i}\right|^{2} & =\sum_{i, j} f_{i} f_{j}\left\langle\varphi^{*} v_{i}, \varphi^{*} v_{j}\right\rangle \\
& =\sum_{i, j} f_{i} f_{j}\left\langle v_{i}, v_{j}\right\rangle \circ \varphi=\sum_{i} f_{i}^{2} \quad \mathfrak{m}_{Y} \text {-a.e. on } \varphi^{-1}(E),
\end{aligned}
$$

and therefore $f_{i}=0 \mathfrak{m}_{\curlyvee}$-a.e. on $\varphi^{-1}(E)$ for every $i=0, \ldots, n-1$.

We now prove that the dimension is constant.

Theorem 4.8 (From parallel transport to constant dimension). Let (X, d, m) be any $\operatorname{RCD}(K, \infty)$ space such that for any Lipschitz test plan $\boldsymbol{\pi}$, any $t \in[0,1]$ and any $\bar{V} \in \mathrm{e}_{t}^{*} L^{2}(T \mathrm{X})$, there exists the parallel transport $V$ along $\boldsymbol{\pi}$ such that $V_{t}=\bar{V}$.

Then the tangent module has constant dimension, i.e., in its dimensional decomposition $\left(E_{i}\right)_{i \in \mathbb{N} \cup\{\infty\}}$ one of the $E_{i}$ 's has full measure and the others are negligible.

Proof. We argue by contradiction, thus we shall assume that for some $i, j \in \mathbb{N} \cup$ $\{\infty\}, i \neq j$, we have $\mathfrak{m}\left(E_{i}\right), \mathfrak{m}\left(E_{j}\right)>0$. Let $F_{0} \subset E_{i}$ and $F_{1} \subset E_{j}$ be bounded, with positive and finite measure, consider

$$
\mu_{0}:=\left.\mathfrak{m}\left(F_{0}\right)^{-1} \mathfrak{m}\right|_{F_{0}}, \quad \mu_{1}:=\mathfrak{m}\left(F_{1}\right)^{-1} \mathfrak{m}_{F_{1}},
$$

let $\boldsymbol{\pi}$ be the unique optimal geodesic plan connecting them, and recall that it is a test plan (see [19]). Since $\pi\left(\mathrm{e}_{0}^{-1}\left(F_{0}\right)\right)=\mu_{0}\left(F_{0}\right)=1$ and the dimension of $L^{2}(T \mathrm{X})$ on $F_{0}$ is $i$, by Lemma 4.7 above we see that for the dimensional decomposition $\left(\tilde{E}_{n}^{0}\right)_{n \in \mathbb{N} \cup\{\infty\}}$ of $\mathrm{e}_{0}^{*} L^{2}(T \mathrm{X})$ we have $\boldsymbol{\pi}\left(\tilde{E}_{i}^{0}\right)=1$ and $\boldsymbol{\pi}\left(\tilde{E}_{k}^{0}\right)=0$ for every $k \neq i$. Similarly, for the the dimensional decomposition $\left(\tilde{E}_{n}^{1}\right)_{n \in \mathbb{N} \cup\{\infty\}}$ of $\mathrm{e}_{1}^{*} L^{2}(T \mathrm{X})$ we have $\boldsymbol{\pi}\left(\tilde{E}_{j}^{1}\right)=1$ and $\boldsymbol{\pi}\left(\tilde{E}_{k}^{1}\right)=0$ for every $k \neq j$. In particular we have

$$
\boldsymbol{\pi}\left(\tilde{E}_{i}^{0} \Delta \tilde{E}_{i}^{1}\right)=\boldsymbol{\pi}\left(\tilde{E}_{i}^{0}\right)=1>0 .
$$

Now notice that from basic considerations about optimal transport we have that $\boldsymbol{\pi}$ is concentrated on geodesics starting from $F_{0}$ and ending in $F_{1}$. The constant speed of any such geodesic is bounded from above by $\sup _{x \in F_{0}, y \in F_{1}} \mathrm{~d}(x, y)<\infty$ so that $\pi$ is a Lipschitz test plan. Therefore from Proposition 4.5 we know that the dimensional decomposition of $\mathrm{e}_{t}^{*} L^{2}(T \mathrm{X})$ does not depend on $t$. This however contradicts (4.4), hence the proof is completed.

\subsection{Existence of the parallel transport in a special case}

It is unclear to us whether on general RCD spaces the parallel transport exists. Aim of this section it to show at least that the theory we propose is not empty, i.e., that under suitable assumptions on the space the parallel transport exists. We will not insist in trying to make such assumptions as general as possible (for instance, 
the 'good basis' defined below could consist in different vector fields on different open sets covering our space), as our main concern is only to show that in some circumstances our notion of parallel transport can be shown to exist. We recall a deep deep result about the structure of finite-dimensional RCD spaces: given any $\operatorname{RCD}(K, N)$ space $(\mathrm{X}, \mathrm{d}, \mathfrak{m})$ with $N \in(1, \infty)$ and whose dimensional decomposition is called $\left(A_{k}\right)_{k}$, there exists (a necessarily unique) $n \in \mathbb{N}$ with $n \leq N$ such that $\mathfrak{m}\left(\mathbf{X} \backslash A_{n}\right)=0$. This 'constant dimension' result has been proved by E. Bruè and D. Semola in [5].

We shall work with spaces admitting the following sort of basis for the tangent module.

Definition 4.9 (Good basis). Let $(\mathrm{X}, \mathrm{d}, \mathfrak{m})$ be a given $\operatorname{RCD}(K, N)$ space, for some $K \in \mathbb{R}$ and $N \in(1, \infty)$, which has dimension $n \leq N$. Then a family $\mathcal{W}=$ $\left\{w_{1}, \ldots, w_{n}\right\} \subseteq H_{C}^{1,2}(T \mathbf{X})$ of Sobolev vector fields on $\mathbf{X}$ is said to be a good basis for $L^{2}(T \mathrm{X})$ provided there exists $M>0$ such that the following properties are satisfied:

(i) We have that $w_{1}, \ldots, w_{n}$ constitute a basis for $L^{2}(T \mathbf{X})$ on $\mathbf{X}$ and

$$
\left\{\begin{array}{l}
\left|w_{i}\right| \in\left(M^{-1}, M\right), \\
\left|\left\langle w_{i}, w_{j}\right\rangle\right|<\frac{1}{M^{2} n},
\end{array} \quad \text { m-a.e. in } \mathbf{X}, \quad \text { for every } i, j=1, \ldots, n \text { with } i \neq j .\right.
$$

(ii) We have

$$
\left|\nabla w_{i}\right|_{\mathrm{HS}} \leq M \quad \mathfrak{m} \text {-a.e. in } \mathrm{X}, \quad \text { for every } i=1, \ldots, n \text {. }
$$

Let us notice that the 'hard' assumption here is given by point (ii) - perhaps coupled with the lower bound in (i) -, which imposes an $L^{\infty}$-bound on covariant derivative, while in our setting the $L^{2}$-ones are more natural (compare with Theorem A.2).

Remark 4.10. We notice that the existence proof that we give below in Theorem 4.15 would equally work if existence of good bases is only required locally, i.e., if one requires the existence of an open cover of the space such that on each open set a good basis as above exists. Still, even with this generalisation, having a good basis would be an extremely strong requirement and not really satisfactory (as said in the introduction, we are mainly interested in showing that our notion of parallel transport is non-empty), thus we will avoid pushing in this direction and simply concentrate on the key ideas.

Remark 4.11. Let $\left(M^{n}, g\right)$ be a compact Riemannian manifold of class $C^{1,1}$. Observe that the metric measure space $\left(M, \mathrm{~d}_{g}, \mathrm{Vol}\right)$ is an $\operatorname{RCD}(K, n)$ space for some $K \in \mathbb{R}$, where $\mathrm{d}_{g}$ stands for the length distance induced by the metric $g$ and $\mathrm{Vol}$ is the Riemannian volume measure. Therefore it is straightforward to show - by working with charts - that the tangent module $L^{2}(T M)$ admits a good basis in the sense of Definition 4.9 and Remark 4.10 above. The $C^{1,1}$-regularity assumption is needed in order to build vector fields $w_{1}, \ldots, w_{n}$ having bounded covariant derivatives, i.e., satisfying item (ii) of Definition 4.9. 
A sort of converse implication of what discussed in the previous remark holds as well: any space admitting a good basis $w_{1}, \ldots, w_{n}$ resembles a Riemannian manifold of class $C^{1,1}$, the reason being the 'metric tensor coefficients' $g_{i j}=\left\langle w_{i}, w_{j}\right\rangle$ have Lipschitz representatives, as we are going to see in the following result.

Lemma 4.12. Let $(\mathrm{X}, \mathrm{d}, \mathfrak{m})$ be an $\operatorname{RCD}(K, N)$ space of dimension $n$. Suppose that $\mathrm{X}$ admits a good basis $w_{1}, \ldots, w_{n}$ for $L^{2}(T \mathrm{X})$. Then the function $g_{i j}:=$ $\left\langle w_{i}, w_{j}\right\rangle$ is $\mathfrak{m}$-a.e. equivalent to a Lipschitz function for every $i, j=1, \ldots, n$.

Proof. We know from Proposition 3.4.6 in [15] that each function $g_{i j}$ belongs to $W^{1,2}(\mathbf{X})$ and satisfies $\mathrm{d} g_{i j}(\cdot)=\nabla w_{i}\left(\cdot, w_{j}\right)+\nabla w_{j}\left(\cdot, w_{i}\right)$, whence $g_{i j},\left|\mathrm{~d} g_{i j}\right| \in L^{\infty}(\mathfrak{m})$ by (4.5) and (4.6). This grants that $g_{i j}$ admits a Lipschitz representative by the Sobolev-to-Lipschitz property (see [3], [11], [12]), thus proving the statement.

Remark 4.13. It is unclear to us whether under the assumptions of Lemma 4.12 the space must necessarily be a $C^{1,1}$ manifold, but this is likely the case if one further assumes that the vector fields in the good basis also have bounded divergence. Indeed, under this further assumption such vector fields admit a regular Lagrangian flow, and such flows might be used to build charts (see for instance [20] for a construction in this direction). It is outside the scope of this paper to prove this claim.

Let us start the technical work with the following simple lemma.

Lemma 4.14. Let $\mathscr{H}$ be a Hilbert module on $\mathrm{X}$ and $M>1$. Pick $w_{1}, \ldots, w_{k} \in \mathscr{H}$ such that

$$
\left\{\begin{array}{l}
\left|w_{i}\right| \in\left(M^{-1}, M\right), \\
\left|\left\langle w_{i}, w_{j}\right\rangle\right| \leq \frac{1}{M^{2} k},
\end{array} \quad \text { hold } \mathfrak{m} \text {-a.e. in } \mathbf{X}, \quad \text { for every } i, j=1, \ldots, k \text { with } i \neq j .\right.
$$

For $h_{1}, \ldots, h_{k} \in L^{0}(\mathfrak{m})$, put $w:=\sum_{i=1}^{k} h_{i} w_{i} \in \mathscr{H}^{0}$ (the $L^{0}$-completion of $\mathscr{H}$ ). Then it holds

$$
\frac{1}{M^{2} k} \sum_{i=1}^{k}\left|h_{i}\right|^{2} \leq|w|^{2} \leq M^{2} k \sum_{i=1}^{k}\left|h_{i}\right|^{2} \quad \mathfrak{m} \text {-a.e. on } \mathbf{X}
$$

thus in particular $w \in \mathscr{H}$ if and only if $h_{i} \in L^{2}(\mathfrak{m})$ for every $i=1, \ldots, k$.

Proof. For the second in (4.7), we notice that $\left\langle w_{i}, w_{j}\right\rangle \leq M^{2}$ m-a.e. on $\mathbf{X}$ for every $i, j$, thus

$$
|w|^{2}=\left|\sum_{i=1}^{k} h_{i} w_{i}\right|^{2}=\sum_{i, j=1}^{k} h_{i} h_{j}\left\langle w_{i}, w_{j}\right\rangle \leq M^{2} \sum_{i, j=1}^{k}\left(\frac{1}{2}\left|h_{i}\right|^{2}+\frac{1}{2}\left|h_{j}\right|^{2}\right)=M^{2} k \sum_{i=1}^{k}\left|h_{i}\right|^{2}
$$

For the first inequality we recall that $\left|w_{i}\right|>M^{-1}$ and $\left\langle w_{i}, w_{j}\right\rangle \geq-\frac{1}{M^{2} k}$ for $i \neq j$ 
$\mathfrak{m}$-a.e. on $\mathrm{X}$ to deduce

$$
\begin{aligned}
|w|^{2} & =\left|\sum_{i=1}^{k} h_{i} w_{i}\right|^{2}=\sum_{i=1}^{k}\left|h_{i}\right|^{2}\left|w_{i}\right|^{2}+\sum_{i \neq j} h_{i} h_{j}\left\langle w_{i}, w_{j}\right\rangle \\
& \geq \frac{1}{M^{2}} \sum_{i=1}^{k}\left|h_{i}\right|^{2}-\frac{1}{M^{2} k} \sum_{i \neq j}\left|h_{i} h_{j}\right| \\
& \geq \frac{1}{M^{2}} \sum_{i=1}^{k}\left|h_{i}\right|^{2}-\frac{1}{M^{2} k} \sum_{i \neq j}\left(\frac{1}{2}\left|h_{i}\right|^{2}+\frac{1}{2}\left|h_{j}\right|^{2}\right)=\frac{1}{M^{2} k} \sum_{i=1}^{k}\left|h_{i}\right|^{2} .
\end{aligned}
$$

Hence the statement is achieved.

We now prove existence of the parallel transport for the class of those RCD spaces that admit a good basis for their tangent module.

Theorem 4.15 (Existence of the parallel transport). Let $(\mathrm{X}, \mathrm{d}, \mathfrak{m})$ be any $\operatorname{RCD}(K, N)$ space, where $K \in \mathbb{R}$ and $N \in(1, \infty)$, which admits a good basis for $L^{2}(T \mathrm{X})$. Let $\boldsymbol{\pi}$ be a Lipschitz test plan on $\mathrm{X}$ and fix an initial datum $\bar{V} \in \mathrm{e}_{0}^{*} L^{2}(T \mathrm{X})$. Then there exists the parallel transport $V \in \mathscr{H}^{1,2}(\boldsymbol{\pi})$ along $\boldsymbol{\pi}$ such that $V_{0}=\bar{V}$.

Proof. Let us fix a good basis $w_{1}, \ldots, w_{n} \in H_{C}^{1,2}(T \mathbf{X})$ for $L^{2}(T \mathbf{X})$. Put $W_{i, t}:=$ $\mathrm{e}_{t}^{*} w_{i}$. By item (i) of Proposition 3.25 we have that $W_{i} \in \mathscr{H}^{1,2}(\boldsymbol{\pi})$, with

$$
\mathrm{D}_{\boldsymbol{\pi}} W_{i, t}=\operatorname{Cov}_{t}\left(w_{i}\right),
$$

and therefore from (3.11), (4.6) and the assumption that $\boldsymbol{\pi}$ is Lipschitz we get

$$
\left|\mathrm{D}_{\boldsymbol{\pi}} W_{i, t}\right| \leq M \mathrm{~L}(\boldsymbol{\pi}) .
$$

Also, by the defining property of the pullback map and from (4.5), we have that for every $t \in[0,1]$ it holds

$$
\left\{\begin{array}{l}
\left|W_{i, t}\right| \in\left(M^{-1}, M\right), \\
\left|\left\langle W_{i, t}, W_{j, t}\right\rangle\right| \leq\left(M^{2} n\right)^{-1}, \quad \pi \text {-a.e., } \quad \text { for every } i, j=1, \ldots, n \text { with } i \neq j,
\end{array}\right.
$$

thus Lemma 4.14 grants that there are functions $\bar{g}_{1}, \ldots, \bar{g}_{n} \in L^{2}(\boldsymbol{\pi})$ such that $\bar{V}=\sum_{i=1}^{n} \bar{g}_{i} \mathrm{e}_{0}^{*} w_{i}$. A similar argument applied to the pullback of the map e (recall Proposition 3.9 and the definition (3.7)) and based on the bound (4.8) shows that there are functions $H_{i, j} \in L^{\infty}\left(\boldsymbol{\pi} \times \mathcal{L}_{1}\right)$ such that

$$
\mathrm{D}_{\boldsymbol{\pi}} W_{i, t}=\sum_{j} H_{i, j, t} W_{j, t} \quad \text { a.e. } t .
$$

It will be technically convenient to fix once and for all Borel representatives, still denoted by $H_{i, j}$, of these functions such that

$$
\sup _{\gamma, t}\left|H_{i, j, t}(\gamma)\right|=\left\|H_{i, j}\right\|_{L^{\infty}\left(\boldsymbol{\pi} \times \mathcal{L}_{1}\right)}, \quad \forall i=1, \ldots, n .
$$


We shall look for a parallel transport of the form $V:=\sum_{i} g_{i} W_{i}$ with $g_{i} \in$ $A C^{2}\left([0,1], L^{2}(\boldsymbol{\pi})\right)$. Notice that Lemma 4.14 grants that any such $V$ belongs to $\mathscr{H}^{1,2}(\boldsymbol{\pi})$, with

$$
\begin{aligned}
\mathrm{D}_{\boldsymbol{\pi}} V_{t} & \stackrel{(3.22)}{=} \sum_{i=1}^{n} g_{i, t}^{\prime} W_{i, t}+\sum_{i=1}^{n} g_{i, t} \mathrm{D}_{\boldsymbol{\pi}} W_{i, t} \stackrel{(4.9)}{=} \sum_{i=1}^{n} g_{i, t}^{\prime} W_{i, t}+\sum_{i, j=1}^{n} g_{i, t} H_{i, j, t} W_{j, t} \\
& =\sum_{i=1}^{n}\left(g_{i, t}^{\prime}+\sum_{j=1}^{n} H_{j, i, t} g_{j, t}\right) W_{i, t} \quad \text { for } \mathcal{L}^{1} \text {-a.e. } t \in[0,1] .
\end{aligned}
$$

Hence our $V$ is the desired parallel transport if and only if the functions $g_{1}, \ldots, g_{n}$ solve the system

$$
\left\{\begin{aligned}
g_{i, 0} & =\bar{g}_{i}, & \forall i & =1, \ldots, n, \\
g_{i, t}^{\prime}+\sum_{j=1}^{n} H_{j, i, t} g_{j, t} & =0, & \forall i & =1, \ldots, n \text { and for } \mathcal{L}^{1} \text {-a.e. } t \in[0,1] .
\end{aligned}\right.
$$

To solve this system we shall apply Theorem 2.6 to the Banach (in fact Hilbert) space $\mathbb{B}:=\left[L^{2}(\boldsymbol{\pi})\right]^{n}$ equipped with the norm

$$
\|f\|_{\mathbb{B}}^{2}:=\sum_{i=1}^{n} \int\left|f_{i}\right|^{2} \mathrm{~d} \boldsymbol{\pi}, \quad \forall f=\left(f_{1}, \ldots, f_{n}\right) \in \mathbb{B} .
$$

For every $t \in[0,1]$, define $\lambda_{t} \in \operatorname{End}(\mathbb{B})$ as

$$
\left(\lambda_{t} f\right)_{i}:=-\sum_{j=1}^{n} H_{j, i, t} f_{j}, \quad \forall i=1, \ldots, n \text { and } f=\left(f_{1}, \ldots, f_{n}\right) \in \mathbb{B},
$$

so that the system (4.11) can be rewritten as

$$
\left\{\begin{array}{l}
g_{0}=\bar{g} \\
g_{t}^{\prime}=\lambda_{t} g_{t} \quad \text { for a.e. } t \in[0,1]
\end{array}\right.
$$

where $\bar{g}:=\left(\bar{g}_{1}, \ldots, \bar{g}_{n}\right)$. Theorem 2.6 grants that a solution in $\operatorname{LIP}([0,1], \mathbb{B}) \subset$ $A C^{2}([0,1], \mathbb{B})$ exists provided the $\lambda_{t}$ 's are equibounded and $t \mapsto \lambda_{t} f$ is strongly measurable for every $f \in \mathbb{B}$. The former follows from

$$
\begin{aligned}
\left\|\lambda_{t} f\right\|_{\mathbb{B}}^{2} & =\sum_{i=1}^{n}\left\|\sum_{j=1}^{n} H_{j, i, t} f_{j}\right\|_{L^{2}(\boldsymbol{\pi})}^{2} \leq n \sum_{i, j=1}^{n}\left\|H_{j, i, t} f_{j}\right\|_{L^{2}(\boldsymbol{\pi})}^{2} \\
& \leq n \max _{i, j, t}\left\|H_{j, i, t}\right\|_{L^{\infty}(\boldsymbol{\pi})}^{2} \sum_{i, j=1}^{n}\left\|f_{j}\right\|_{L^{2}(\boldsymbol{\pi})}^{2} \stackrel{(4.10)}{\leq} n^{2} \max _{i, j}\left\|H_{i, j}\right\|_{L^{\infty}\left(\boldsymbol{\pi} \times \mathcal{L}_{1}\right)}^{2}\|f\|_{\mathbb{B}}^{2} .
\end{aligned}
$$

For the latter, notice that since $\mathbb{B}$ is separable it is sufficient to prove that for any $f \in \mathbb{B}$ the map $t \mapsto \lambda_{t} f \in \mathbb{B}$ is weakly measurable. Since $\mathbb{B}$ is also Hilbert we need to show that for every $f, g \in \mathbb{B}$ the map $t \mapsto\left\langle\lambda_{t} f, g\right\rangle_{\mathbb{B}} \in \mathbb{R}$ is measurable. Since we have

$$
\left\langle\lambda_{t} f, g\right\rangle_{\mathbb{B}}=-\sum_{i, j=1}^{n} \int H_{j, i, t} f_{j} g_{i} \mathrm{~d} \boldsymbol{\pi},
$$

the conclusion follows from Fubini's theorem. 


\section{A. Sobolev basis of the tangent module}

In this appendix we show that one can always build a basis of the tangent module of a RCD space which has at least Sobolev regularity, as opposed to just $L^{2}$ regularity. The basic idea used in the construction is based on the observation that 'being a basis' is a non-linear requirement. Technically speaking, the crucial argument is contained in the following lemma.

Lemma A.1. Let $(\mathbf{X}, \mathrm{d}, \mathfrak{m})$ be a $\operatorname{RCD}(K, \infty)$ space and $w \in L^{2}(T \mathbf{X})$. Then there exists $v \in H_{C}^{1,2}(T \mathrm{X})$ such that $\langle v, w\rangle \neq 0$ holds $\mathfrak{m}$-a.e. on $\{|w| \neq 0\}$.

Proof. We can assume $w \neq 0$ or otherwise there is nothing to prove; then replacing if necessary $w$ with $\left(\chi_{\{|w| \leq 1\}}+\chi_{\{|w|>1\}}|w|^{-1}\right) w$ we can also assume that $|w| \leq 1$ $\mathfrak{m}$-a.e. . Let $\left(w_{n}\right) \subset \operatorname{TestV}(\mathbf{X})$ be $L^{2}(T \mathbf{X})$-converging to $w$ and $\tilde{\mathfrak{m}}$ a Borel probability measure on $\mathbf{X}$ such that $\mathfrak{m} \ll \tilde{\mathfrak{m}} \leq \mathfrak{m}$. Then $\left\langle w_{n}, w\right\rangle \rightarrow|w|^{2}$ in $L^{2}(\mathbf{X}, \tilde{\mathfrak{m}})$ and thus

$$
m_{n}:=\tilde{\mathfrak{m}}\left(\left\{\left|\left\langle w_{n}, w\right\rangle\right|>0\right\}\right) \rightarrow m_{\infty}:=\tilde{\mathfrak{m}}(\{|w|>0\})
$$

We now observe that

for every $v, \tilde{w} \in L^{2}(T \mathrm{X})$ and $a>0$ there is $b \in(0, a)$ such that

$$
\tilde{\mathfrak{m}}(\{|\langle\tilde{w}, w\rangle|>0\} \cap\{\langle v+b \tilde{w}, w\rangle=0\})=0 .
$$

Indeed, putting for brevity $E_{b}:=\{|\langle\tilde{w}, w\rangle|>0\} \cap\{\langle v+b \tilde{w}, w\rangle=0\}$, we have

$$
\tilde{\mathfrak{m}}\left(E_{b} \cap E_{b^{\prime}}\right) \leq \tilde{\mathfrak{m}}\left(\{|\langle\tilde{w}, w\rangle|>0\} \cap\left\{\left(b-b^{\prime}\right)\langle\tilde{w}, w\rangle=0\right\}\right)=0, \quad \forall b \neq b^{\prime},
$$

so that the claim follows from the finiteness of $\tilde{\mathfrak{m}}$ and the fact that the interval $(0, a)$ is uncountable.

Now put $\alpha_{n}:=\left\|\left|w_{n}\right|\right\|_{L^{\infty}(\mathrm{X})}+\left\|w_{n}\right\|_{W_{C}^{1,2}(T \mathrm{X})}$ and recursively define decreasing sequences $\left(\beta_{n}\right),\left(\gamma_{n}\right) \subset(0, \infty)$ such that $\beta_{1}=1$ and for every $n \in \mathbb{N}$ we have

$$
\begin{aligned}
& 3 \beta_{n+1} \leq \gamma_{n+1} \leq \beta_{n} \\
& \text { and for } E_{n}:=\left\{\left|\left\langle\sum_{i=1}^{n} \frac{\beta_{i}}{\alpha_{i}} w_{i}, w\right\rangle\right| \geq \gamma_{n+1}\right\} \text {, it holds } \tilde{\mathfrak{m}}\left(E_{n}\right) \geq \frac{m_{n}}{1+1 / n} .
\end{aligned}
$$

To see that this is possible, let $\beta_{1}=1$, and notice that trivially $\left\{\left|\left\langle\frac{\beta_{1}}{\alpha_{1}} w_{1}, w\right\rangle\right|>0\right\}=$ $\left\{\left|\left\langle w_{1}, w\right\rangle\right|>0\right\}$, so that for $\gamma_{2} \in\left(0, \beta_{1}\right)$ sufficiently small the above holds. Now assume that $\beta_{n-1}$ and $\gamma_{n}$ have been found, use (A.2) for $v:=\sum_{i=1}^{n-1} \frac{\beta_{i}}{\alpha_{i}} w_{i}, \tilde{w}:=w_{n}$ and $a:=\gamma_{n} / 3$ to find $\beta_{n}:=b<\gamma_{n} / 3$ such that $\tilde{\mathfrak{m}}\left(\left\{\left|\left\langle\sum_{i=1}^{n} \frac{\beta_{i}}{\alpha_{i}} w_{i}, w\right\rangle\right|>0\right\}\right)$ $\geq \tilde{\mathfrak{m}}\left(\left\{\left|\left\langle w_{n}, w\right\rangle\right|>0\right\}\right)=m_{n}$. Hence for $\gamma_{n+1} \in\left(0, \beta_{n}\right)$ sufficiently small the claim holds.

We claim that the vector $v:=\sum_{i \geq 1} \frac{\beta_{i}}{\alpha_{i}} w_{i}$ satisfies the conclusion of the statement, and start observing that $\beta_{i} \leq 3^{-i}$, and thus

$$
\left\|\frac{\beta_{i}}{\alpha_{i}} w_{i}\right\|_{W_{C}^{1,2}(T \mathrm{X})} \leq 3^{-i}\left\|\alpha_{i}{ }^{-1} w_{i}\right\|_{W_{C}^{1,2}(T \mathrm{X})} \leq 3^{-i}
$$


by definition of $\alpha_{i}$. Hence the series converges in $W_{C}^{1,2}(T \mathbf{X})$, so that $v$ is well defined and belongs to $H_{C}^{1,2}(T \mathrm{X})$. Now notice that by construction and (A.1) we have $\tilde{\mathfrak{m}}\left(E_{n}\right) \rightarrow m_{\infty}$ and $\tilde{\mathfrak{m}}\left(E_{n} \backslash\{|w|>0\}\right)=0$, so that $\tilde{\mathfrak{m}}\left(\{|w|>0\} \backslash \cup_{n} E_{n}\right)=0$. Hence to conclude it is sufficient to show that for every $n \geq 1$ it holds $\langle v, w\rangle \neq 0$ $\tilde{\mathfrak{m}}$-a.e. on $E_{n}$. Fix such $n$, let $m>n$, and observe that by definition of the $\alpha_{i}$ 's and $\beta_{i}$ 's we have

$$
\left|\left\langle\frac{\beta_{m}}{\alpha_{m}} w_{m}, w\right\rangle\right| \leq 3^{n-m+1} \beta_{n+1}\left|\left\langle\alpha_{m}^{-1} w_{m}, w\right\rangle\right| \leq 3^{n-m+1} \beta_{n+1}, \quad \tilde{\mathfrak{m}} \text {-a.e. },
$$

so that

$$
\left|\sum_{m>n}\left\langle\frac{\beta_{m}}{\alpha_{m}} w_{m}, w\right\rangle\right| \leq \frac{3}{2} \beta_{n+1} \leq \frac{1}{2} \gamma_{n+1} .
$$

On the other hand by construction we have that $\left|\sum_{i=1}^{n}\left\langle\frac{\beta_{i}}{\alpha_{i}} w_{i}, w\right\rangle\right| \geq \gamma_{n+1}$ holds $\tilde{\mathfrak{m}}$-a.e. on $E_{n}$, granting that $|\langle v, w\rangle| \geq \frac{1}{2} \gamma_{n+1} \tilde{\mathfrak{m}}$-a.e. on $E_{n}$.

By repeatedly applying Lemma A.1, we can find a family of $H_{C}^{1,2}(T X)$-Sobolev generators of the tangent module on any $\operatorname{RCD}(K, \infty)$ space $\mathrm{X}$, as follows.

Theorem A.2 (Sobolev basis of the tangent module). Let $(\mathrm{X}, \mathrm{d}, \mathfrak{m})$ be an $\operatorname{RCD}(K, \infty)$ space, for some constant $K \in \mathbb{R}$. Suppose that the dimensional decomposition of $\mathrm{X}$ is given by $\left(A_{n}\right)_{n \in \mathbb{N}}$. Then there exists a sequence of vector fields $\left(v_{n}\right)_{n \geq 1} \subseteq$ $H_{C}^{1,2}(T \mathrm{X})$ such that

$$
v_{1}, \ldots, v_{n} \text { is a basis for } L^{2}(T \mathrm{X}) \text { on } A_{n}, \quad \text { for every } n \in \mathbb{N}^{+} .
$$

Proof. The thesis can be equivalently rewritten in the following way:

$$
v_{1}, \ldots, v_{n} \text { are independent on } \bigcup_{k \geq n} A_{k}, \quad \text { for every } n \in \mathbb{N}^{+} \text {. }
$$

We build the sequence $\left(v_{n}\right)_{n}$ by means of a recursive argument. First of all, choose a vector field $w \in L^{2}(T \mathrm{X})$ such that $0<|w| \leq 1 \mathrm{~m}$-a.e. in $\bigcup_{k \geq 1} A_{k}$, then pick $v_{1} \in H_{C}^{1,2}(T \mathrm{X})$ such that $\left\langle v_{1}, w\right\rangle \neq 0$ m-a.e. in $\bigcup_{k>1} A_{k}$, whose existence is granted by Lemma A.1. Thus in particular we have $\left.\mid v_{1}\right\rceil>0$ m-a.e. in $\bigcup_{k \geq 1} A_{k}$, proving (A.3) for $n=1$. Now suppose to have already found $v_{1}, \ldots, v_{n}$ satisfying the required property. It can be easily seen that there exists $w \in L^{2}(T \mathrm{X})$ such that $\left\langle v_{1}, w\right\rangle=\cdots=\left\langle v_{n}, w\right\rangle=0$ and $0<|w| \leq 1$ hold $\mathfrak{m}$-a.e. in the set $\bigcup_{k>n} A_{k}$. Hence Lemma A.1 ensures the existence of a vector field $v_{n+1} \in H_{C}^{1,2}(T \mathrm{X})$ such that $\left\langle v_{n+1}, w\right\rangle \neq 0$ m-a.e. in $\bigcup_{k>n} A_{k}$.

Now take any $f_{1}, \ldots, f_{n+1} \in L^{\infty}(\mathfrak{m})$ such that $\sum_{i=1}^{n+1} f_{i} v_{i}=0 \mathfrak{m}$-a.e. in $\bigcup_{k>n} A_{k}$, thus one has $f_{n+1}\left\langle v_{n+1}, w\right\rangle=\sum_{i=1}^{n+1} f_{i}\left\langle v_{i}, w\right\rangle=0 \mathfrak{m}$-a.e. in $\bigcup_{k>n} A_{k}$, from which we can deduce that $f_{n+1}=0$ holds $\mathfrak{m}$-a.e. in $\bigcup_{k>n} A_{k}$. Therefore $\sum_{i=1}^{n} f_{i} v_{i}=0$ m-a.e. in $\bigcup_{k>n} A_{k}$ and accordingly also $f_{1}=\ldots=f_{n}=0 \mathfrak{m}$-a.e. in $\bigcup_{k>n} A_{k}$, as a consequence of the independence of $v_{1}, \ldots, v_{n}$. This grants that the vector fields $v_{1}, \ldots, v_{n+1}$ are independent on $\bigcup_{k>n} A_{k}$, proving (A.3) for $n+1$. The thesis is then achieved. 


\section{References}

[1] Ambrosio, L., Colombo, M. And Di Marino, S.: Sobolev spaces in metric measure spaces: reflexivity and lower semicontinuity of slope. In Variational methods for evolving objects, 1-58. Adv. St. in Pure Math. 67, Math. Soc. Japan, 2015.

[2] Ambrosio, L., Gigli, N. and Savaré, G.: Calculus and heat flow in metric measure spaces and applications to spaces with Ricci bounds from below. Invent. Math 195 (2014), no. 2, 289-391.

[3] Ambrosio, L., Gigli, N. And Savaré, G.: Metric measure spaces with Riemannian Ricci curvature bounded from below. Duke Math. J. 163 (2014), no. 7, 1405-1490.

[4] Ambrosio, L., Gigli, N. And Savaré, G.: Bakry-Émery curvature-dimension condition and Riemannian Ricci curvature bounds. Ann. Probab. 43 (2015), no. 1, 339-404.

[5] Bruè, E. And Semola, D. Constancy of the dimension for $\operatorname{RCD}(K, N)$ spaces via regularity of Lagrangian flows. To appear in Comm. Pure Appl. Math.

[6] Brù̀, E. And Semola, D. Regularity of Lagrangian flows over $\operatorname{RCD}^{*}(K, N)$ spaces. To appear in J. Reine Angew. Math.

[7] Caputo, E., Gigli, N. and Pasqualetto, E.: Work in progress..

[8] Colding, T.H. And NABer, A.: Sharp Hölder continuity of tangent cones for spaces with a lower Ricci curvature bound and applications. Ann. of Math. (2) 176 (2012), no. 2, 1173-1229.

[9] De Philippis, G. And Gigli, N. Non-collapsed spaces with Ricci curvature bounded from below. J. Éc. Polytech. Math. 5 (2018), 613-650.

[10] Diestel, J. And UhL, J. J., JR.: Vector measures. Mathematical Surveys 15, American Mathematical Society, Providence, RI, 1977.

[11] GigLi, N.: The splitting theorem in non-smooth context. Preprint, arXiv: 1302.5555, 2013.

[12] GigLi, N.: An overview of the proof of the splitting theorem in spaces with nonnegative Ricci curvature. Anal. Geom. Metr. Spaces 2 (2014), no. 1, 169-213.

[13] GigLi, N.: On the differential structure of metric measure spaces and applications. Mem. Amer. Math. Soc. 236 (2015), no. 1113, vi+91 pp.

[14] Gigli, N.: Lecture notes on differential calculus on RCD spaces. Publ. Res. Inst. Math. Sci. 54 (2018), no. 4, 855-918.

[15] GigLI, N.: Nonsmooth differential geometry - an approach tailored for spaces with Ricci curvature bounded from below. Mem. Amer. Math. Soc. 251 (2018), no. 1196, $\mathrm{v}+161 \mathrm{pp}$.

[16] GigLI, N.: Riemann curvature tensor on RCD spaces and possible applications. C. $R$. Math. Acad. Sci. Paris 357 (2019), no. 7, 613-619.

[17] Gigli, N. and Pasqualetto, E.: Equivalence of two different notions of tangent bundle on rectifiable metric measure spaces. Preprint, arXiv: 1611.09645, 2016.

[18] Gigli, N. And Pasqualetto, E.: Behaviour of the reference measure on RCD spaces under charts. To appear in Comm. Anal. Geom.

[19] Gigli, N., Rajala, T. And Sturm, K.-T.: Optimal maps and exponentiation on finite-dimensional spaces with Ricci curvature bounded from below. J. Geom. Anal. 26 (2016), no. 4, 2914-2929. 
[20] Gigli, N. And Rigoni, C.: Recognizing the flat torus among $\operatorname{RCD}^{*}(0, N)$ spaces via the study of the first cohomology group. Calc. Var. Partial Differential Equations 57 (2018), no. 4, Art. 104, 39 pp.

[21] Kell, M. And Mondino, A.: On the volume measure of non-smooth spaces with Ricci curvature bounded below. Ann. Sc. Norm. Super. Pisa Cl. Sci. (5), 18 (2018), no. 2, 593-610.

[22] Lott, J. And Villani, C.: Ricci curvature for metric-measure spaces via optimal transport. Ann. of Math. (2) 169 (2009), no. 3, 903-991.

[23] Mondino, A. And Naber, A.: Structure theory of metric-measure spaces with lower Ricci curvature bounds. J. Eur. Math. Soc. (JEMS) 21 (2019), no. 6, 1809-1854.

[24] Petrunin, A.: Parallel transportation for Alexandrov space with curvature bounded below. Geom. Funct. Anal. 8 (1998), no. 1, 123-148.

[25] Petrunin, A.: Alexandrov meets Lott-Villani-Sturm. Münster J. Math. 4 (2011), $53-64$.

[26] Sturm, K.-T.: On the geometry of metric measure spaces. I. Acta Math. 196 (2006), no. 1, 65-131.

[27] Sturm, K.-T.: On the geometry of metric measure spaces. II. Acta Math. 196 (2006), no. 1, 133-177.

[28] Villani, C.: Inégalités isopérimétriques dans les espaces métriques mesurés [d'après F. Cavalletti \& A. Mondino]. In Séminaire Bourbaki, 2016/2017. Exposés 1120-1135. Arstérisque 407 (2019), Exp. No. 1127, 213-265. Available at: http://www . bourbaki.ens.fr/TEXTES/1127.pdf.

[29] Villani, C.: Synthetic theory of Ricci curvature bounds. Jpn. J. Math. 11 (2016), no. 2, 219-263.

[30] Zhang, H.-C. And Zhu, X.-P.: Ricci curvature on Alexandrov spaces and rigidity theorems. Comm. Anal. Geom. 18 (2010), no. 3, 503-553.

Received February 28, 2018; revised November 22, 2018.

Published online December 17, 2019.

Nicola Gigli: SISSA, Via Bonomea 265, 34136 Trieste, Italy.

E-mail: ngigli@sissa.it

Enrico Pasqualetto: Department of Mathematics and Statistics, PO Box 35, 40014

University of Jyväskylä, Jyväskylä, Finland.

E-mail: enpasqua@jyu.fi

This research has been supported by the MIUR SIR-grant 'Nonsmooth Differential Geometry' (RBSI147UG4). 